\title{
Minimal/Measurable Residual Disease Monitoring in NPM1-Mutated Acute Myeloid Leukemia: A Clinical Viewpoint and Perspectives
}

\author{
Fabio Forghieri ${ }^{1, *}$, Patrizia Comoli ${ }^{2,3}$, Roberto Marasca ${ }^{1}$, Leonardo Potenza ${ }^{1}$ and Mario Luppi ${ }^{1}$ \\ 1 Department of Medical and Surgical Sciences, Section of Hematology, University of Modena and Reggio \\ Emilia, Azienda Ospedaliero-Universitaria di Modena, Via del Pozzo 71, 41124 Modena, Italy; \\ roberto.marasca@unimore.it (R.M.); leonardo.potenza@unimore.it (L.P.); mario.luppi@unimore.it (M.L.) \\ 2 Pediatric Hematology/Oncology Unit, Istituto di Ricovero e Cura a Carattere Scientifico (IRCCS) Policlinico \\ San Matteo, Viale Golgi 19, 27100 Pavia, Italy; pcomoli@smatteo.pv.it \\ 3 Cell Factory, Istituto di Ricovero e Cura a Carattere Scientifico (IRCCS) Policlinico San Matteo, \\ Viale Golgi 19, 27100 Pavia, Italy \\ * Correspondence: fabio.forghieri@unimore.it; Tel.: +39-059-4222447; Fax: +39-059-4222386
}

Received: 15 October 2018; Accepted: 3 November 2018; Published: 6 November 2018

\begin{abstract}
Acute myeloid leukemia (AML) with NPM1 gene mutations is currently recognized as a distinct entity, due to its unique biological and clinical features. We summarize here the results of published studies investigating the clinical application of minimal/measurable residual disease (MRD) in patients with NPM1-mutated AML, receiving either intensive chemotherapy or hematopoietic stem cell transplantation. Several clinical trials have so far demonstrated a significant independent prognostic impact of molecular MRD monitoring in NPM1-mutated AML and, accordingly, the Consensus Document from the European Leukemia Net MRD Working Party has recently recommended that NPM1-mutated AML patients have MRD assessment at informative clinical timepoints during treatment and follow-up. However, several controversies remain, mainly with regard to the most clinically significant timepoints and the MRD thresholds to be considered, but also with respect to the optimal source to be analyzed, namely bone marrow or peripheral blood samples, and the correlation of MRD with other known prognostic indicators. Moreover, we discuss potential advantages, as well as drawbacks, of newer molecular technologies such as digital droplet PCR and next-generation sequencing in comparison to conventional RQ-PCR to quantify NPM1-mutated MRD. In conclusion, further prospective clinical trials are warranted to standardize MRD monitoring strategies and to optimize MRD-guided therapeutic interventions in NPM1-mutated AML patients.
\end{abstract}

Keywords: NPM1-mutated acute myeloid leukemia; molecular minimal/measurable residual disease monitoring; prognostic thresholds and timepoints; intensive chemotherapy; allogeneic hematopoietic stem cell transplantation; clinical outcome

\section{Introduction}

The results of cytogenetic and molecular examinations at diagnosis of non-promyelocytic acute myeloid leukemia (AML), combined with the achievement of morphologic complete remission (CR) after remission induction chemotherapy, have significant prognostic impact on clinical outcome and usually serve to guide post-remission therapeutic strategies in younger adult patients [1-3]. However, the morphologic assessment of bone marrow (BM) blast percentage by light microscopy is significantly hampered by limited sensitivity and inter-observer variability, so that it is generally recognized that, when $5 \%$ myeloblasts is considered to be the cut-off to define morphologic $\mathrm{CR}$, about $10^{10}$ leukemic 
cells may still persist in the patient [3,4]. The risk stratification in AML patients actually remains inadequate, and there is growing interest in the use of more sensitive laboratory tools, such as multiparametric flow cytometry (MFC) or molecular techniques, to detect low levels of residual disease in either BM or peripheral blood (PB) at different treatment timepoints [1]. The evaluation of minimal residual disease, also referred to as measurable residual disease (MRD), is considered useful to more precisely define AML response to intensive chemotherapy, thereby refining risk stratification [1]. Some studies have demonstrated that MRD persistence in a condition of morphologic CR confers high relapse risk and adverse prognosis, comparable to the one associated with persisting leukemic cells at microscopic examinations [4-6]. Interestingly, the recently revised European Leukemia Net (ELN) recommendations for AML management have introduced the category $C R$ without $M R D\left(\mathrm{CR}_{\mathrm{MRD}-}\right)$ [7]. Moreover, long-term MRD monitoring in patients in CR may serve to early detect leukemia relapse [1]. MRD assessment captures the diversities of the underlying cytogenetic/genetic AML characteristics and also recapitulates patients' heterogeneity regarding chemotherapy bioavailability, metabolism and resistance, thus resulting in a unique in vivo tool to evaluate chemosensitivity of leukemic cells $[4,8,9]$. Moreover, even within homogeneous genetic subgroups, the long-term outcome of AML patients depends on the clearance of the molecular lesion, as clearly demonstrated in other myeloid neoplasms, namely chronic myeloid leukemia and acute promyelocytic leukemia [1,4]. Beyond MFC, several molecular techniques are currently available for MRD determination in AML patients, including real-time quantitative polymerase chain reaction (RQ-PCR), which is highly sensitive, reliable, rapid and reproducible between different laboratories, as well as newer but less standardized tools such as next-generation sequencing (NGS) and digital droplet PCR (ddPCR) [2,4]. RQ-PCR allows MRD detection in patients with documentation of chimeric fusion genes generated by balanced chromosomal rearrangements, especially in cases of acute promyelocytic leukemia and core-binding factor (CBF) leukemias, but also in AML cases with other genetic alterations, such as insertions/duplications (e.g., NPM1, FLT3-ITD, MLL-PTD), point mutations (CEBPA, IDH1/2, KIT, RAS, RUNX1, TP53) or gene overexpression (WT1, EVI1, ERG) [1,2,4]. While MFC, despite its limitations, could enable MRD assessment in the vast majority of AML patients, a major drawback of RQ-PCR is its applicability only to those cases, accounting for approximately $50-60 \%$ of younger AML patients, who bear at least one molecular lesion, specific and stable over the treatment course, which could reliably be monitored using this molecular technique $[2,4,10]$.

\section{NPM1 Mutations in AML: Biological and Clinical Features}

Nucleophosmin (NPM1) gene encodes for a protein which physiologically shuttles between nucleus and cytoplasm, acting as a molecular chaperone to establish multiple protein-protein interactions [11]. NPM1 protein is normally involved in critical cell functions, such as control of ribosome formation and export, stabilization of the oncosuppressor $\mathrm{p} 14^{\mathrm{Arf}}$ protein in the nucleolus, and regulation of centrosome duplication [12-14]. NPM1 gene mutations, occurring in approximately $30 \%$ of adult AML cases, and in $50-60 \%$ of AML cases with normal karyotype, represent one of the most frequent molecular lesions observed in AML [14,15]. Since the discovery of NPM1 mutations in 2005 by Falini et al. [16], more than 55 different mutations, mainly occurring in the exon 12 of the gene, have been described, but three mutation types (A, B and D) account for $95 \%$ of all cases $[8,14,17]$. NPM1 gene mutations result in structural changes of the C-terminus of NPM1 protein, with subsequent aberrant cytoplasmic delocalization, leading to a unique immunohistochemical pattern detectable on BM trephine biopsy $[16,18]$. This cytoplasmic accumulation of NPM1-mutated protein causes perturbations of multiple cellular pathways through a combination of loss of functions and gain of functions, critical for leukemogenesis [12-14,17]. Notably, it was recently reported that NPM1-mutated protein dislocated PU.1 into cytoplasm with it, whereas CEBPA and RUNX1, the master transcription factors that collaborate with PU.1 to activate granulo-monocytic lineage-fates, remained in the nucleus. However, without nuclear PU.1, their coregulator interactions were toggled from coactivators to corepressors, thus repressing $>500$ granulocyte and monocyte terminal differentiation genes [19]. 
As expected for founder genetic lesions, NPM1 mutations are specific, being almost exclusively restricted to AML, usually de novo, and generally expressed in the whole leukemic population $[13,14,20]$. Notably, NPM1-mutated AML, showing distinctive genetic, pathologic, immunophenotypic and clinical features, has now been recognized as a full distinct entity among AML with recurrent genetic abnormalities in the 2016 revision of World Health Organization (WHO) classification of myeloid neoplasms and acute leukemia [21]. While the infrequent presence of coexisting chromosomal abnormalities, observed in only $15 \%$ of patients, does not appear to modify the prognostic effects of NPM1 mutations [7,22,23], prognosis may be significantly influenced by accompanying molecular lesions, mainly FLT3 and DNMT3A gene mutations, documented in about $40 \%$ and $50 \%$ of NPM1-mutated AML cases, respectively $[7,15,17]$. In detail, the better risk outcomes observed in NPM1-mutated AML patients were generally considered limited to cases without concurrent FLT3-ITD mutations [17,24]. Furthermore, the deleterious prognostic effects of FLT3-ITD have previously been found to be most clinically relevant when co-occurring with NPM1 and DNMT3A mutations, as opposed with either mutation alone [25]. However, recent studies have suggested that patients with NPM1 mutation and FLT3-ITD with a low $(<0.5)$ allelic ratio have a similar favorable outcome as patients with NPM1-mutated AML without FLT3-ITD [26-29]. Thus, both of these latter groups are currently considered favorable according to the 2017 ELN risk stratification, in contrast to NPM1-mutated AML associated with FLT3-ITD with high allelic ratio, which is characterized by higher relapse rate and poorer overall survival (OS) [7].

\section{ELN Recommendations for MRD Assessment}

Based upon the above-mentioned biological and clinical characteristics, especially their homogeneous mutation pattern in AML patients, NPM1 mutations may be considered an ideal leukemia-specific target for MRD detection [2]. Since the first application by Gorello et al. of sensitive and specific RQ-PCR assays as a reliable system to quantitatively assess NPM1-mutated gene copies [30], several studies have investigated the clinical implications of MRD monitoring in NPM1-mutated AML undergoing intensive therapeutic approaches (Table 1) [30-73]. 
Table 1. MRD monitoring in NPM1-mutated AML series: review of the literature.

\begin{tabular}{|c|c|c|c|c|c|c|}
\hline $\begin{array}{l}\text { Reference/Type of } \\
\text { Study }\end{array}$ & $\begin{array}{c}\text { Number of Patients/Median } \\
\text { Age (Years, Range) }\end{array}$ & $\begin{array}{l}\text { Number of Samples } \\
\text { (PB/BM) }\end{array}$ & $\begin{array}{l}\text { Number of Samples per } \\
\text { Patient (Median, Range) }\end{array}$ & $\begin{array}{c}\text { Molecular } \\
\text { Method/Material }\end{array}$ & NPM1 Mutation Type & $\begin{array}{l}\text { Sensitivity of the } \\
\text { Assay }\end{array}$ \\
\hline $\begin{array}{l}\text { Gorello et al., } \\
2006[30] / \text { retrospective }\end{array}$ & 20/NA & $\begin{array}{l}\mathrm{NA} \text { (PB and/or BM } \\
\text { samples at diagnosis } \\
\text { and/or at different } \\
\text { timepoints) }\end{array}$ & $\begin{array}{l}\text { NA (13 patients analyzed at } \\
\text { diagnosis and } \\
\text { post-induction). MRD } \\
\text { kinetics during follow-up of } \\
3 \text { representative patients } \\
\text { is reported }\end{array}$ & $\begin{array}{l}\text { RQ-PCR/cDNA ( } 5 \text { cases), } \\
\text { DNA (15 cases) }\end{array}$ & $\begin{array}{c}\text { A, B (cDNA); A, B, D, E, } \\
\text { G, H (DNA) }\end{array}$ & $10^{-3}-10^{-6}$ \\
\hline $\begin{array}{c}\text { Chou et al., } \\
2007[31] / \text { retrospective }\end{array}$ & $38 / 47(17-87)$ & $194 \mathrm{BM}$ & 5 & RQ-PCR/DNA & 7 different mutations & $10^{-5}$ \\
\hline $\begin{array}{l}\text { Papadaki et al., } \\
2008 \text { [32]/retrospective }\end{array}$ & $51 / 58(22-78)$ & 154 (18 PB/136 BM) & $\begin{array}{l}\text { NA (26 patients analyzed at } \\
\text { diagnosis and at least at } \\
2 \text { timepoints during therapy; } \\
27 \text { patients analyzed at } \\
\text { diagnosis and after } \\
\text { induction therapy) }\end{array}$ & RQ-PCR/cDNA & A & $10^{-5}$ \\
\hline $\begin{array}{l}\text { Barragan et al., } \\
2008[33] /-\end{array}$ & $24 / 17$ cases $(71 \%)<60$ yeas & 97 (5 PB/92 BM) & NA & RQ-PCR/cDNA & A & $10^{-5}$ \\
\hline $\begin{array}{c}\text { Bacher et al., } \\
2009[34] / \text { retrospective }\end{array}$ & 13/47 (20-66) & 139 (PB/BM) & $7(2-25)$ & RQ-PCR/DNA & $\mathrm{A}, \mathrm{B}$ & $10^{-4}-10^{-6}$ \\
\hline $\begin{array}{c}\text { Schnittger et al., } \\
2009[35] / \text { retrospective }\end{array}$ & $252 / 59(20-79)$ & $\begin{array}{c}1227 \text { (28 PB at } \\
\text { diagnosis/1199 BM) }\end{array}$ & $4(2-16)$ & RQ-PCR/cDNA & 17 different mutations & $10^{-4}-10^{-6}$ \\
\hline $\begin{array}{c}\text { Stahl et al., } \\
2010[36] / \text { retrospective }\end{array}$ & 25/53 (21-73) & 76 (38 PB/38 BM) & $1-2$ & RQ-PCR/DNA & A & $10^{-4}-10^{-6}$ \\
\hline $\begin{array}{l}\text { Dvorakova et al., } \\
2010 \text { [37]/retrospective }\end{array}$ & 25/51 (43-75) & 1026 (339 PB/687 BM) & $28(11-68)$ & RQ-PCR/DNA & 9 different mutations & $10^{-4}-10^{-6}$ \\
\hline $\begin{array}{c}\text { Ommen et al., } \\
2010[38] / \text { retrospective }\end{array}$ & 180 (54 in HR)/NA & $\begin{array}{l}193 \mathrm{CCR} \text { and } 70 \text { relapse } \\
\text { samples }\end{array}$ & NA & RQ-PCR/cDNA & NA & $10^{-4}-10^{-6}$ \\
\hline $\begin{array}{c}\text { Kristensen et al., } \\
2011[39] / \text { retrospective }\end{array}$ & 20/61 (41-76) & 204 & NA & RQ-PCR/DNA & A & $2.4 \times 10^{-5}$ \\
\hline $\begin{array}{c}\text { Kronke et al., } \\
2011[40] / \text { retrospective }\end{array}$ & $245 / 49(19-61)$ & 1682 (410 PB/1272 BM) & NA & RQ-PCR/cDNA & 6 different mutations & $10^{-5}-10^{-6}$ \\
\hline $\begin{array}{c}\text { Thol et al., } \\
2012[41] / \text { retrospective }\end{array}$ & 10/NA (adult patients) & 45 & NA & $\begin{array}{l}\text { NGS, RQ-PCR/DNA, } \\
\text { cDNA }\end{array}$ & $\begin{array}{l}3 \text { different mutations } \\
(8 \text { cases } A, 1 \text { case } D \\
1 \text { case atypical })\end{array}$ & $10^{-4}$ \\
\hline
\end{tabular}


Table 1. Cont.

\begin{tabular}{|c|c|c|c|c|c|c|}
\hline $\begin{array}{l}\text { Reference/Type of } \\
\text { Study }\end{array}$ & $\begin{array}{c}\text { Number of Patients/Median } \\
\text { Age (Years, Range) }\end{array}$ & $\begin{array}{c}\text { Number of Samples } \\
\text { (PB/BM) }\end{array}$ & $\begin{array}{l}\text { Number of Samples per } \\
\text { Patient (Median, Range) }\end{array}$ & $\begin{array}{c}\text { Molecular } \\
\text { Method/Material }\end{array}$ & NPM1 Mutation Type & $\begin{array}{c}\text { Sensitivity of the } \\
\text { Assay }\end{array}$ \\
\hline $\begin{array}{l}\text { Abdelhamid et al., } \\
2012 \text { [42]/retrospective }\end{array}$ & $20 / 55(27-69)$ & $\begin{array}{c}116 \text { (20 at diagnosis, } \\
96 \text { follow-up samples, } \\
\text { namely } 55 \text { PB and } \\
41 \mathrm{BM})\end{array}$ & $4.5(2-11)$ & RQ-PCR/DNA & $\begin{array}{l}3 \text { different mutations } \\
(\mathrm{A}, \mathrm{B}, \text { insCACG) }\end{array}$ & $10^{-4}-10^{-5}$ \\
\hline $\begin{array}{l}\text { Schiller et al., } \\
2012[43] / \text { retrospective }\end{array}$ & $\begin{array}{c}30 \text { (among } 54 \text { FLT3-ITD+ } \\
\text { patients)/62 }\end{array}$ & NA & NA & RQ-PCR/cDNA & NA & $10^{-4}-10^{-6}$ \\
\hline $\begin{array}{c}\text { Shayegi et al., } \\
2013[44] / \text { retrospective }\end{array}$ & $155 / 51(20-79)$ & 1750 (817 PB/933 BM) & NA & RQ-PCR/DNA & A, B, D & $10^{-5}$ \\
\hline $\begin{array}{c}\text { Jeziskova et al., } \\
2013[45] / \text { retrospective }\end{array}$ & $\begin{array}{l}6 \text { (among } 8 \text { patients with } \\
\text { IDH2 mutations)/57 (53-72) }\end{array}$ & $60(17$ PB / 43 BM) & $3-14$ & RQ-PCR/DNA & A, B & $10^{-4}-10^{-6}$ \\
\hline $\begin{array}{l}\text { Salipante et al., } \\
2014[46] / \text { retrospective }\end{array}$ & $6 / \mathrm{NA}$ & $22 \mathrm{BM}$ & $2-6$ & NGS/DNA & $\begin{array}{c}\text { No need for } \\
\text { mutation-specific probes }\end{array}$ & $10^{-5}$ \\
\hline $\begin{array}{l}\text { Hubmann et al., } \\
2014[47] / \text { retrospective }\end{array}$ & $158 / 57(18-80)$ & $588 \mathrm{BM}$ & NA & RQ-PCR/cDNA & A, B, D & $10^{-6}$ \\
\hline $\begin{array}{c}\text { Bacher et al., } \\
2014 \text { [48]/retrospective }\end{array}$ & $99 / \mathrm{NA}$ & 498 & $4(1-28)$ & digital PCR/cDNA & 37 different mutations & $10^{-4}-10^{-5}$ \\
\hline $\begin{array}{l}\text { Lambert et al., } \\
2014 \text { [49]/prospective }\end{array}$ & $\begin{array}{l}77 \text { patients with NPM1 } \\
\text { mutation/61 (57-65) }\end{array}$ & 250 (125 PB/125 BM) & NA & RQ-PCR/cDNA & A, B, D & $10^{-5}$ \\
\hline $\begin{array}{c}\text { Debarri et al., } \\
2015 \text { [50]/retrospective }\end{array}$ & $31 / 60(23-70)$ & 94 & NA & $\begin{array}{c}\text { RQ-PCR for NPM1; NGS } \\
\text { for IDH1/2 and } \\
D N M T 3 A / c D N A\end{array}$ & A, B, D & $10^{-5}$ \\
\hline $\begin{array}{l}\text { Pettersson et al., } \\
2016[51] /-\end{array}$ & $19 / 64(28-78)$ & $63(2 \mathrm{~PB} / 61 \mathrm{BM})$ & $1-9$ & RQ-PCR/DNA & $\mathrm{A}$ & $10^{-5}$ \\
\hline $\begin{array}{c}\text { Karas et al., } \\
2016[52] / \text { retrospective }\end{array}$ & $60 / 54(30-66)$ & $60 \mathrm{BM}$ & 1 (pre-HSCT) & RQ-PCR/cDNA & A, B, D & NA \\
\hline $\begin{array}{l}\text { Alizad Ghandforoush } \\
\text { et al., } \\
2016 \text { [53]/retrospective }\end{array}$ & $11 / 42(28-63)$ & $71(\mathrm{~PB} / \mathrm{BM})$ & NA & RQ-PCR/DNA & $\mathrm{A}$ & $10^{-5}$ \\
\hline $\begin{array}{c}\text { Ivey et al., } \\
2016 \text { [54]/prospective }\end{array}$ & $\begin{array}{c}346 \text { in preliminary } \\
\text { development phase, } 91 \text { in } \\
\text { validation cohort } / 50(6-68)\end{array}$ & $\begin{array}{l}2569 \text { in first phase } \\
(1667 \mathrm{~PB} / 902 \mathrm{BM})\end{array}$ & 6 & RQ-PCR/cDNA & 27 different mutations & $10^{-5}$ \\
\hline
\end{tabular}


Table 1. Cont.

\begin{tabular}{|c|c|c|c|c|c|c|}
\hline $\begin{array}{l}\text { Reference/Type of } \\
\text { Study }\end{array}$ & $\begin{array}{c}\text { Number of Patients/Median } \\
\text { Age (Years, Range) }\end{array}$ & $\begin{array}{c}\text { Number of Samples } \\
\text { (PB/BM) }\end{array}$ & $\begin{array}{l}\text { Number of Samples per } \\
\text { Patient (Median, Range) }\end{array}$ & $\begin{array}{c}\text { Molecular } \\
\text { Method/Material }\end{array}$ & NPM1 Mutation Type & $\begin{array}{c}\text { Sensitivity of the } \\
\text { Assay }\end{array}$ \\
\hline $\begin{array}{c}\text { Kayser et al., } \\
2016[55] / \text { retrospective }\end{array}$ & $67 / 55(21-70)$ & $\begin{array}{l}406(\mathrm{BM}) \text { at different } \\
\text { timepoints }\end{array}$ & $\begin{array}{c}\text { pre-transplant MRD was } \\
\text { available for } 39 / 51(76.4 \%) \\
\text { patients in CR at HSCT } \\
\text { (22 cases, } 56 \% \text { MRD-positive) }\end{array}$ & RQ-PCR/cDNA & A, B, D & $10^{-5}-10^{-6}$ \\
\hline $\begin{array}{c}\text { Malmberg et al., } \\
2017[56] / \text { retrospective }\end{array}$ & $\begin{array}{c}17(3 \text { NPM1-mutated } \\
\text { patients)/39 }(2-71)\end{array}$ & NA & NA & RQ-PCR, NGS/DNA & A & $10^{-5}$ \\
\hline $\begin{array}{c}\text { Balsat et al., } \\
2017[57] / \text { retrospective }\end{array}$ & $152 / 49(21-61)$ & $\begin{array}{c}304(\mathrm{~PB} / \mathrm{BM}) \text { at } \\
\text { diagnosis and } 270 \\
\text { post-induction } \\
(135 \mathrm{~PB} / 135 \mathrm{BM})\end{array}$ & $\begin{array}{l}\text { samples obtained at } \\
\text { diagnosis and after } \\
\text { induction CHT }\end{array}$ & RQ-PCR/cDNA & A, B, D & $10^{-5}$ \\
\hline $\begin{array}{l}\text { Schieppati et al., } \\
2017 \text { [58]/- }\end{array}$ & $68 / 56(27-74)$ & NA & $\begin{array}{c}4 \text { PB/BM samples (at } \\
\text { diagnosis, TP1 at CR, TP2 } \\
\text { post-consolidation, TP3 post } \\
\text { 1st cycle of Ara-C) }\end{array}$ & RQ-PCR/cDNA & NA & $10^{-4}-10^{-5}$ \\
\hline $\begin{array}{l}\text { Mencia-Trinchant et al., } \\
2017 \text { [59]/- }\end{array}$ & $3 /-$ & NA & $\begin{array}{l}\text { Sequential determination of } \\
\text { MRD levels }\end{array}$ & $\mathrm{ddPCR} / \mathrm{cDNA}$ & $\begin{array}{c}\text { multiplex assay effective } \\
\text { in a range of diverse } \\
\text { common and rare NPM1 } \\
\text { mutations (14) }\end{array}$ & $10^{-4}-10^{-5}$ \\
\hline $\begin{array}{c}\text { Getta et al., } \\
2017[60] / \text { retrospective }\end{array}$ & $\begin{array}{l}104 \text { (10 with NPM1 } \\
\text { mutation)/58 (21-78) }\end{array}$ & $\begin{array}{l}58 \mathrm{BM} \text { at diagnosis, } \\
83 \mathrm{BM} \text { before HSCT } \\
\text { for NGS }\end{array}$ & NA & NGS, MFC/DNA & 2 different mutations & $10^{-4}$ \\
\hline $\begin{array}{c}\text { Bill et al., } \\
2018[61] / \text { retrospective }\end{array}$ & $51 / 62(33-74)$ & 51 (40 PB/11 BM) & $\begin{array}{l}\text { samples collected directly } \\
\text { before HSCT }\end{array}$ & $\mathrm{ddPCR} / \mathrm{cDNA}$ & A, D & $10^{-4}$ \\
\hline $\begin{array}{l}\text { Jongen-Lavrencic et al., } \\
2018 \text { [62]/retrospective }\end{array}$ & $\begin{array}{l}430 \text { (168 with NPM1 } \\
\text { mutation)/51 (18-66) }\end{array}$ & $\begin{array}{l}482 \mathrm{~PB} / \mathrm{BM} \text { samples at } \\
\text { diagnosis, } 430 \mathrm{BM} \\
\text { samples after treatment }\end{array}$ & 2 (at diagnosis and in CR) & NGS, MFC/DNA & NA & $10^{-4}$ \\
\hline $\begin{array}{c}\text { Zhou et al., } \\
2018[63] / \text { retrospective }\end{array}$ & $59 / 57(21-79)$ & $104 \mathrm{BM}$ & pre-HSCT and post-HSCT & NGS, MFC/DNA & - & $10^{-4}$ \\
\hline $\begin{array}{l}\text { Zappasodi et al., } \\
\text { 2018 [64]/retrospective, } \\
\text { real-life study }\end{array}$ & $\begin{array}{l}201 \text { (116 with NPM1 } \\
\text { mutation)/58 }\end{array}$ & NA & $\begin{array}{l}\text { Availability of samples } \\
\text { during treatment and } \\
\text { follow-up was variable. }\end{array}$ & RQ-PCR/cDNA & NA & $10^{-4}-10^{-5}$ \\
\hline
\end{tabular}


Table 1. Cont.

\begin{tabular}{|c|c|c|c|c|c|c|}
\hline $\begin{array}{l}\text { Reference/Type of } \\
\text { Study }\end{array}$ & $\begin{array}{c}\text { Number of Patients/Median } \\
\text { Age (Years, Range) }\end{array}$ & $\begin{array}{c}\text { Number of Samples } \\
\text { (PB/BM) }\end{array}$ & $\begin{array}{l}\text { Number of Samples per } \\
\text { Patient (Median, Range) }\end{array}$ & $\begin{array}{c}\text { Molecular } \\
\text { Method/Material }\end{array}$ & NPM1 Mutation Type & $\begin{array}{c}\text { Sensitivity of the } \\
\text { Assay }\end{array}$ \\
\hline $\begin{array}{l}\text { Delsing Malmberg et al., } \\
2018 \text { [65]/retrospective }\end{array}$ & $29 / 49(18-66)$ & 83 (6 PB/77 BM) & $\begin{array}{c}3 \text { (at diagnosis, before and } \\
\text { after HSCT) }\end{array}$ & NGS/DNA & $\begin{array}{l}\text { all recurrent insertion } \\
\text { mutations in NPM1 exon } \\
12 \text { (mutation A in } \\
25 \text { cases) }\end{array}$ & $10^{-4}$ \\
\hline $\begin{array}{l}\text { Kapp-Schwoerer et al., } \\
2018 \text { [66]/retrospective }\end{array}$ & $611 / 18-60$ & $\begin{array}{l}6339(2812 \mathrm{~PB} / \\
3527 \mathrm{BM})\end{array}$ & $\begin{array}{l}\text { NA (samples analyzed at } \\
\text { diagnosis, during treatment } \\
\text { and follow-up) }\end{array}$ & RQ-PCR/cDNA & NA & $10^{-5}-10^{-6}$ \\
\hline $\begin{array}{c}\text { Caprioli et al., } \\
2018[67] / \text { retrospective }\end{array}$ & $27 / 57(23-65)$ & $27 \mathrm{BM}$ & 1 (pre alloHSCT) & RQ-PCR/cDNA & NA & $10^{-4}$ \\
\hline $\begin{array}{c}\text { Patkar et al., } \\
2018[68] / \text { retrospective }\end{array}$ & $83 / \mathrm{NA}$ & NA & NA & NGS/DNA & 12 different mutations & $10^{-5}$ \\
\hline $\begin{array}{c}\text { Onecha et al., } \\
2018 \text { [69]/retrospective }\end{array}$ & $\begin{array}{c}63 \text { (57 with NPM1 } \\
\text { mutation)/54 (42-66) }\end{array}$ & $\begin{array}{c}106 \text { BM (51 after } \\
\text { induction, } 55 \text { post } \\
\text { consolidation CHT) }\end{array}$ & 2 & NGS/DNA & A & $10^{-5}$ \\
\hline $\begin{array}{c}\text { Petrova et al., } \\
2018[70] / \text { retrospective }\end{array}$ & $\begin{array}{c}90(22 \text { positive for } I D H 1 / 2 \\
\text { mutations, } 11 \text { with } N P M 1 \\
\text { mutation)/ } 61(22-82)\end{array}$ & $149 \mathrm{BM}$ & $\begin{array}{l}\text { NA (90 at diagnosis, } 22 \text { after } \\
\text { induction, } 37 \text { during } \\
\text { follow-up) }\end{array}$ & $\begin{array}{l}\text { RQ-PCR for NPM1 } \\
\text { mutations/cDNA }\end{array}$ & A & NA \\
\hline $\begin{array}{c}\text { Prata et al., } \\
2018[71] / \text { retrospective }\end{array}$ & $\begin{array}{l}\text { 34 with newly diagnosed } \\
\text { NPM1-mutated AML/77 } \\
\text { (55-85) }\end{array}$ & $\begin{array}{c}\text { MRD assessment } \\
\text { available on BM } \\
\text { samples in } 6 \text { patients }\end{array}$ & NA & RQ-PCR/NA & A, B, D & NA \\
\hline $\begin{array}{c}\text { Ottone et al., } \\
2018[72] / \text { retrospective }\end{array}$ & $\begin{array}{l}556 \text { de novo AML (177 with } \\
\text { NPM1 mutation)/49 (16-89) }\end{array}$ & $\begin{array}{l}\text { NA (BM samples at } \\
\text { diagnosis, during } \\
\text { follow-up, at relapse) }\end{array}$ & $\begin{array}{l}\text { NA (NPM1-mutated } \\
\text { transcripts monitoring in } \\
51 \text { cases) }\end{array}$ & RQ-PCR/cDNA & $\mathrm{A}$ & $10^{-5}$ \\
\hline $\begin{array}{c}\text { Gaksch et al., } \\
2018[73] / \text { retrospective }\end{array}$ & $\begin{array}{l}34 \text { cytogenetically normal } \\
\text { AML (16 cases with NPM1 } \\
\text { mutation)/47 (22-79) }\end{array}$ & $\begin{array}{c}34 \mathrm{BM} \text { or } \mathrm{PB} \text { at } \\
\text { diagnosis, } 27 \mathrm{BM} \\
\text { samples in remission }\end{array}$ & $\begin{array}{c}2 \text { (at diagnosis and after at } \\
\text { least one } \\
\text { consolidation therapy) }\end{array}$ & $\begin{array}{l}\text { NGS/DNA extracted } \\
\text { from BM slides }\end{array}$ & $\begin{array}{l}\text { Multiplex analysis of } \\
19 \text { genes, including } \\
\text { NPM1 mutational } \\
\text { hotspots }\end{array}$ & $10^{-2}$ \\
\hline
\end{tabular}

MRD, minimal/measurable residual disease; AML, acute myeloid leukemia; PB, peripheral blood; BM, bone marrow; NA, not available; RQ-PCR, real-time quantitative polymerase chain

reaction; cDNA, complimentary DNA; HR, hematological relapse; CCR, continuous complete remission; NGS, next-generation sequencing; HSCT, hematopoietic stem cell transplantation;

CR, complete remission; TP, timepoint; ddPCR, digital droplet PCR; MFC, multiparametric flow cytometry; CHT, chemotherapy. 
The consensus document from the ELN MRD Working Party indicates that AML patients with NPM1 mutations, such as patients with RUNX1-RUNX1T1, CBFb-MYH11 or PML-RARA fusion transcripts, should have molecular MRD assessment at informative clinical timepoints. During the active treatment phase, MRD assessment for these molecular lesions is recommended at minimum at diagnosis, after 2 cycles of induction/consolidation chemotherapy, and at the end of treatment [74]. Furthermore, after the end of treatment, samples for MRD analyses should in general be collected every 3 months for 24 months. Thereafter, monitoring beyond 2 years of follow-up should be based on the relapse risk of the patient and decided individually [74]. Specifically regarding NPM1-mutated AML, with or without other concomitant mutations, monitoring of NPM1 transcripts is recommended in $\mathrm{BM}$ and $\mathrm{PB}$, if possible. If NPM1-mutated MRD remains negative in PB but positive in BM after the end of treatments, transcripts should be closely monitored every 4 weeks for at least 3 months, in order to evaluate any MRD increase. Conversely, if a rising MRD is not confirmed or MRD becomes undetectable, then MRD retesting may be regularly performed at 3-month intervals for at least the first 2 years after the end of treatment [74].

At first glance, the clinical management of NPM1-mutated AML patients according to these recommendations could appear clearly defined. However, several controversial issues remain, especially with regard to the most clinically significant timepoints and MRD thresholds to be considered, but also with respect to the best source to be analyzed, namely BM or PB samples, and correlation of MRD with other known prognostic indicators (Table 2) [8]. 
Table 2. Clinical impact of MRD monitoring in NPM1-mutated AML patients.

\begin{tabular}{|c|c|c|c|c|c|c|c|}
\hline Reference & $\begin{array}{c}\text { Intensive } \\
\text { CHT/HSCT } \\
\text { (No. of Patients) }\end{array}$ & $\begin{array}{l}\text { Significant MRD } \\
\text { Threshold }\end{array}$ & Prognostic Timepoints & $\begin{array}{l}\text { Correlation with } \\
\text { Other Molecular } \\
\text { Markers }\end{array}$ & Clonal Evolution & $\begin{array}{c}\text { Median Time } \\
\text { since Molecular } \\
\text { to Morphologic } \\
\text { Relapse (Range) }\end{array}$ & Clinical Relevance \\
\hline $\begin{array}{l}\text { Gorello et al., } \\
2006 \text { [30] }\end{array}$ & Yes/NA & $\begin{array}{l}\text { NA ( } 3 / 5 \text { cases with MRD } \\
<1 \% \text { long-term survivors) }\end{array}$ & NA & NA & NA & NA & $\begin{array}{l}\text { NPM1-mutated copies closely } \\
\text { correlated with clinical status } \\
\text { and predicted impending } \\
\text { hematologic relapse in } 2 \text { cases }\end{array}$ \\
\hline $\begin{array}{l}\text { Chou et al., } \\
2007[31]\end{array}$ & $\begin{array}{l}38 \mathrm{Yes} / 11 \\
\text { alloHSCT }\end{array}$ & $0.1 \%$ & $\begin{array}{l}\text { End of consolidation; } \\
\text { follow-up }\end{array}$ & $\begin{array}{c}\text { FLT3-ITD } \\
\text { worsened RFS }\end{array}$ & No & $\begin{array}{l}4.9 \text { months } \\
(1-12.3)\end{array}$ & $\begin{array}{ll}- & \begin{array}{l}\text { Any rise of mutant signals } \\
\text { during follow-up increased } \\
\text { relapse risk }\end{array} \\
\text { MRD }<0.1 \% \text { predicted longer } \\
\text { RFS and OS } \\
\text { - } \quad \text { Failure to achieve } 2 \text { logs } \\
\text { reduction after consolidation } \\
\text { predicted shorter RFS and OS }\end{array}$ \\
\hline $\begin{array}{l}\text { Papadaki et al., } \\
2008 \text { [32] }\end{array}$ & $\begin{array}{l}50 \mathrm{Yes} / 11 \\
\text { alloHSCT }\end{array}$ & $\begin{array}{l}\text { NA (median } \log _{10} \\
\text { reduction of } 2.48 \text { post } \\
\text { induction correlated with } \\
\text { response to therapy) }\end{array}$ & NA & NA & $\begin{array}{c}\text { In } 2 / 21 \text { relapses } \\
(9.5 \%) \text { NPM1 } \\
\text { mutation was lost. }\end{array}$ & NA & $\begin{array}{l}\text { In selected patients, it was } \\
\text { possible to correlate the } \\
\text { changes of the NPM1 mutation } \\
\text { A levels with the clinical course } \\
\text { of the disease }\end{array}$ \\
\hline $\begin{array}{l}\text { Barragan et al., } \\
\quad 2008[33]\end{array}$ & 24 Yes/NA & $\begin{array}{l}\text { NA (in } 19 \text { patients in CCR } \\
\text { a median 3\% MRD after } \\
\text { induction was shown. } \\
\text { Median MRD level after } \\
\text { consolidation } 0.3 \%\end{array}$ & NA & $\begin{array}{l}\text { Expression levels } \\
\text { of } W T 1 \text { and } N P M 1 \\
\text { showed strong } \\
\text { positive } \\
\text { correlation. }\end{array}$ & No & $\begin{array}{l}\text { MRD increase } 1 \text { to } \\
5 \text { months before } \\
\text { relapse in } 4 / 6 \\
\text { cases }\end{array}$ & $\begin{array}{l}\text { MRD negativity or } \\
\text { maintenance of very low levels } \\
\text { of NPM1 mutation in patients } \\
\text { in continuous CR } \\
\text { Increase in NPM1 transcripts } \\
\text { before or at the time of relapse }\end{array}$ \\
\hline $\begin{array}{l}\text { Bacher et al., } \\
2009[34]\end{array}$ & $-/ 13$ alloHSCT & NA & $\begin{array}{l}\text { All } 4 \text { patients }(29 \%) \text { with } \\
\text { persistent MRD positive } \\
\text { after HSCT relapsed }\end{array}$ & $\begin{array}{l}\text { Correlation with } \\
\text { molecular } \\
\text { chimerism. }\end{array}$ & No & 24 days (12-38) & $\begin{array}{ll}\text { - } & \text { After HSCT } 10 / 14 \text { cases }(71 \%) \\
\text { PCR-negative, of which } 4 \\
\text { achieved stable CR. } \\
\text { MRD increase preceded } \\
\text { morphologic relapse }\end{array}$ \\
\hline $\begin{array}{l}\text { Schnittger et al., } \\
2009 \text { [35] }\end{array}$ & $\begin{array}{l}252 \text { Yes/53 } \\
\text { alloHSCT }\end{array}$ & $\begin{array}{l}0.01 \% \text { during } 1 \mathrm{st} \text { line } \\
\text { treatment. } 0.1 \% \text { after } \\
\text { HSCT and during 2nd } \\
\text { line treatment. }\end{array}$ & $\begin{array}{ll}- & \begin{array}{l}\text { early assessment } \\
\text { (days 18-60) }\end{array} \\
- & \text { days 60-121 } \\
- & \text { days 121-365 } \\
- & \text { longer than 1 year } \\
\text { after start } \\
\text { of treatment }\end{array}$ & $\begin{array}{c}\text { FLT3-ITD } \\
\text { prognostic factor } \\
\text { affecting EFS }\end{array}$ & No & 62 days (15-221) & $\begin{array}{l}\text { MRD for NPM1 mutation } \\
\text { assessed at } 4 \text { different time } \\
\text { intervals after start of therapy } \\
\text { (mainly after day 60) is an } \\
\text { independent and highly } \\
\text { predictive parameter for EFS. }\end{array}$ \\
\hline
\end{tabular}


Table 2. Cont

\begin{tabular}{|c|c|c|c|c|c|c|c|}
\hline Reference & $\begin{array}{c}\text { Intensive } \\
\text { CHT/HSCT } \\
\text { (No. of Patients) }\end{array}$ & $\begin{array}{l}\text { Significant MRD } \\
\text { Threshold }\end{array}$ & Prognostic Timepoints & $\begin{array}{l}\text { Correlation with } \\
\text { Other Molecular } \\
\text { Markers }\end{array}$ & Clonal Evolution & $\begin{array}{c}\text { Median Time } \\
\text { since Molecular } \\
\text { to Morphologic } \\
\text { Relapse (Range) } \\
\end{array}$ & Clinical Relevance \\
\hline $\begin{array}{l}\text { Stahl et al., } \\
2010[36]\end{array}$ & -/25 alloHSCT & NA & Post-HSCT follow-up & $\begin{array}{l}\text { High rate of } \\
\text { congruent results } \\
\text { with chimerism } \\
\text { analysis }\end{array}$ & No & NA & $\begin{array}{l}\text { - Concordant results in BM and } \\
\text { PB in } 60 \% \text { of sample pairs. } \\
\text { Cases with }<0.01 \% \text { MRD in BM } \\
\text { were negative in PB. } \\
\text { Higher MRD levels in BM } \\
(>0.2 \%) \text { predicted PB positivity }\end{array}$ \\
\hline $\begin{array}{l}\text { Dvorakova et al., } \\
2010[37]\end{array}$ & $\begin{array}{l}25 \text { Yes } / 4 \\
\text { alloHSCT }\end{array}$ & $\begin{array}{l}\text { Reappearance of NPM1 } \\
\text { mutation or one order } \\
\text { NCN increase in patients } \\
\text { with persistent positivity } \\
\text { at any timepoint. }\end{array}$ & NA & NA & No & 97 days (12-141) & $\begin{array}{l}\text { Molecular relapse preceded } \\
\text { hematological relapse in } 80 \% \text { of } \\
\text { evaluable patients. } \\
\text { Strong correlation between PB } \\
\text { and BM samples }\end{array}$ \\
\hline $\begin{array}{l}\text { Ommen et al., } \\
2010 \text { [38] }\end{array}$ & NA/NA & $\begin{array}{c}0.005 \% \text { (threshold to } \\
\text { define molecular relapse) }\end{array}$ & NA & $\begin{array}{l}\text { More rapid MRD } \\
\text { growth in } \\
\text { FLT3-ITD+ cases }\end{array}$ & No & $\begin{array}{l}120 \text { days without } \\
\text { FLT3-ITD, } 65 \text { days } \\
\text { in FLT3-ITD+ cases }\end{array}$ & 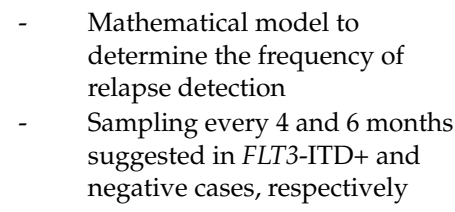 \\
\hline $\begin{array}{l}\text { Kristensen et al., } \\
2011 \text { [39] }\end{array}$ & $20 \mathrm{Yes} / \mathrm{NA}$ & NA & $\begin{array}{c}\text { Reoccurrence of NPM1 } \\
\text { mutation at any time } \\
\text { was associated with } \\
\text { relapse. }\end{array}$ & $\begin{array}{l}\text { NPM1 mutation is } \\
\text { superior to WT1 } \\
\text { expression levels } \\
\text { as MRD marker. }\end{array}$ & $\begin{array}{c}\text { No (NPM1 } \\
\text { mutation stability. } \\
\text { Karyotype } \\
\text { evolution in } 56 \% \\
\text { of relapses). }\end{array}$ & 46 days (20-182) & $\begin{array}{l}\text { - All relapses were associated } \\
\text { with high levels of } \\
\text { NPM1 mutation } \\
\text { Detectable NPM1 mutation } \\
\text { following a CR period was } \\
\text { accompanied by morphologic } \\
\text { relapse in all cases }\end{array}$ \\
\hline $\begin{array}{l}\text { Kronke et al., } \\
2011[40]\end{array}$ & $\begin{array}{l}245 \mathrm{Yes} / 80 \\
\text { alloHSCT }\end{array}$ & $2 \%$ & $\begin{array}{ll}- & \text {-MRD negativity } \\
& \text { after } 2 \\
\text { induction cycles } \\
-\quad & \text {-After completion } \\
\text { of therapy } \\
\text { - } \quad \text {-During follow-up }\end{array}$ & $\begin{array}{l}\text { FLT3-ITD } \\
\text { significant factor } \\
\text { for inferior } \\
\text { survival. }\end{array}$ & $\begin{array}{c}\text { In } 5 \text { patients } \\
\text { NPM1 mutation } \\
\text { was not detectable } \\
\text { at relapse (9\% of } \\
\text { evaluable relapse } \\
\text { samples) }\end{array}$ & $\begin{array}{l}2.6 \text { months } \\
(0.4-23.6)\end{array}$ & 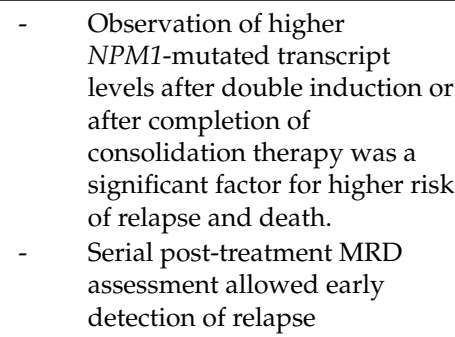 \\
\hline
\end{tabular}


Table 2. Cont.

\begin{tabular}{|c|c|c|c|c|c|c|c|}
\hline Reference & $\begin{array}{c}\text { Intensive } \\
\text { CHT/HSCT } \\
\text { (No. of Patients) }\end{array}$ & $\begin{array}{l}\text { Significant MRD } \\
\text { Threshold }\end{array}$ & Prognostic Timepoints & $\begin{array}{l}\text { Correlation with } \\
\text { Other Molecular } \\
\text { Markers }\end{array}$ & Clonal Evolution & $\begin{array}{c}\text { Median Time } \\
\text { since Molecular } \\
\text { to Morphologic } \\
\text { Relapse (Range) }\end{array}$ & Clinical Relevance \\
\hline $\begin{array}{l}\text { Thol et al., } \\
2012 \text { [41] }\end{array}$ & 10 Yes/NA & $\begin{array}{c}\mathrm{NA} \\
\text { (mean allelic ratio at } \\
\text { diagnosis 0.37, } \\
\text { range 0.29-0.46) }\end{array}$ & NA & NA & $\begin{array}{l}\text { NPM1 mutation } \\
\text { not detectable in } \\
1 / 4 \text { patients at } \\
\text { relapse. }\end{array}$ & NA & $\begin{array}{l}\text { - Parallel assessment of MRD by } \\
\text { NGS and RQ-PCR was } \\
\text { concordant in } 95 \% \text { of } \\
\text { analyzed samples } \\
\text { NGS as a potentially highly } \\
\text { flexible and reliable tool to } \\
\text { assess MRD }\end{array}$ \\
\hline $\begin{array}{l}\text { Abdelhamid } \\
\text { et al., } 2012 \text { [42] }\end{array}$ & 20 Yes/NA & NA & After induction therapy & $\begin{array}{l}\text { Similar kinetics of } \\
\text { FLT3-ITD, NPM1 } \\
\text { and WT1 } \\
\text { expression for } \\
\text { predicted clinical } \\
\text { trend. }\end{array}$ & No & $\begin{array}{l}\text { NA for NPM1 } \\
\text { mutation }\end{array}$ & $\begin{array}{l}\text { - The } 3 \text { MRD markers tested } \\
\text { showed comparable kinetics in } \\
17 / 20(85 \%) \text { cases }\end{array}$ \\
\hline $\begin{array}{l}\text { Schiller et al., } \\
2012 \text { [43] }\end{array}$ & $\begin{array}{l}54 \text { (30 NPM1- } \\
\text { mutated) Yes/7 } \\
\text { alloHSCT }\end{array}$ & NA & $\begin{array}{l}\text { NA (samples collected } \\
\text { at diagnosis, during } \\
\text { treatment and } \\
\text { follow-up) }\end{array}$ & $\begin{array}{l}\text { MRD for FLT3-ITD } \\
\text { as sensitive as } \\
\text { other MRD } \\
\text { parameters as } \\
\text { NPM1 mutations } \\
\text { or MLL-PTD } \\
\end{array}$ & NA & NA & $\begin{array}{ll}\text { - } & \text { MRD negativity predicted } \\
\text { lasting remission independent } \\
\text { of alloHSCT or non-alloHSCT } \\
\text { - } \quad \text { FLT3-ITD analyses equivalently } \\
\text { sensitive in PB samples }\end{array}$ \\
\hline $\begin{array}{c}\text { Shayegi et al., } \\
2013 \text { [44] }\end{array}$ & $\begin{array}{l}155 \text { Yes } / 40 \\
\text { alloHSCT }\end{array}$ & $\begin{array}{c}\text {-MRD level }>1 \% \text { after } \\
\text { conventional CHT-MRD } \\
\text { level }>10 \% \text { after alloHSCT }\end{array}$ & $\begin{array}{l}\text { After intensive CHT } \\
\text { and after HSCT }\end{array}$ & $\begin{array}{l}\text { Prognostic role of } \\
\text { MRD remained } \\
\text { significant after } \\
\text { adjustment for } \\
\text { FLT3-ITD status }\end{array}$ & No & $\begin{array}{ll}- & 121 \text { days } \\
& (70-172) \text { for } \\
\text { MRD }>1 \% \\
\text { 66 days } \\
(34-98) \text { for } \\
\text { MRD }>10 \%\end{array}$ & $\begin{array}{l}\text { - } \quad \text { Rising of MRD was associated } \\
\text { with increased risk of relapse. } \\
\text { DFS and OS analyses revealed } \\
\text { significantly worse outcomes in } \\
\text { patients with rising MRD levels }\end{array}$ \\
\hline $\begin{array}{c}\text { Jeziskova et al., } \\
2013 \text { [45] }\end{array}$ & $\begin{array}{l}8 \mathrm{Yes} / 4 \\
\text { alloHSCT }\end{array}$ & NA & NA & $\begin{array}{l}\text { Concordance of } \\
\text { quantitative } \\
\text { detection of } I D H 2 \\
\text { and NPM1 } \\
\text { mutations, except } \\
\text { for on case. }\end{array}$ & NA & NA & $\begin{array}{l}\text { - In } 5 / 6 \text { patients, the kinetics of } \\
\text { IDH2 quantification was nearly } \\
\text { identical to the kinetics of } \\
\text { NPM1 mutations }\end{array}$ \\
\hline
\end{tabular}


Table 2. Cont.

\begin{tabular}{|c|c|c|c|c|c|c|c|}
\hline Reference & $\begin{array}{c}\text { Intensive } \\
\text { CHT/HSCT } \\
\text { (No. of Patients) }\end{array}$ & $\begin{array}{l}\text { Significant MRD } \\
\text { Threshold }\end{array}$ & Prognostic Timepoints & $\begin{array}{l}\text { Correlation with } \\
\text { Other Molecular } \\
\text { Markers }\end{array}$ & Clonal Evolution & $\begin{array}{c}\text { Median Time } \\
\text { since Molecular } \\
\text { to Morphologic } \\
\text { Relapse (Range) }\end{array}$ & Clinical Relevance \\
\hline $\begin{array}{l}\text { Salipante et al., } \\
2014 \text { [46] }\end{array}$ & $6 \mathrm{Yes} / \mathrm{NA}$ & NA & NA & NA & $\begin{array}{l}\text { In } 2 \text { patients } \\
\text { genetically distinct } \\
\text { NPM1 tumor } \\
\text { subclones were } \\
\text { detected }\end{array}$ & NA & $\begin{array}{l}\text { As a proof of principle, NGS } \\
\text { documented MRD in all } \\
\text { samples deemed negative by } \\
\text { flow cytometry, without the } \\
\text { need for } \\
\text { mutation-specific probes }\end{array}$ \\
\hline $\begin{array}{l}\text { Hubmann et al., } \\
2014 \text { [47] }\end{array}$ & $\begin{array}{l}158 \text { Yes } / 30 \\
\text { alloHSCT }\end{array}$ & $\begin{array}{l}\text { cut-off ratio } 0.01 \text { and 3-log } \\
\text { reduction }\end{array}$ & After induction $\mathrm{CHT}$ & $\begin{array}{l}\text { Prognostic role of } \\
\text { MRD regardless of } \\
\text { ELN risk } \\
\text { stratification. }\end{array}$ & $\begin{array}{l}\text { NPM1 mutation } \\
\text { not detectable in } 3 \\
\text { of } 45(6.7 \%) \\
\text { patients at relapse }\end{array}$ & 58 days (20-98) & $\begin{array}{l}\text { Assessment of MRD levels after } \\
\text { induction was significant to } \\
\text { identify patients in CR with } \\
\text { high risk of relapse. There was } \\
\text { also a trend for OS }\end{array}$ \\
\hline $\begin{array}{l}\text { Bacher et al., } \\
2014 \text { [48] }\end{array}$ & 99 Yes/NA & $0.01 \%$ & $\begin{array}{ll}- & \text { days } 18-60 \\
- & \text { days } 61-120 \\
- & \text { days } 121-365 \\
- & >\text { day } 365\end{array}$ & NA & No & NA & $\begin{array}{ll}\text { - } & \text { NPM1 mutation levels by } \\
\text { digital and conventional } \\
\text { RQ-PCR significantly } \\
\text { correlated a diagnosis and } \\
\text { during follow-up } \\
\text { digital PCR threshold of } 0.01 \% \\
\text { prognostically relevant. }\end{array}$ \\
\hline $\begin{array}{l}\text { Lambert et al., } \\
2014 \text { [49] }\end{array}$ & 77 Yes/NA & $0.1 \%$ (in BM samples) & $\begin{array}{ll}- & \text { after } \\
\text { induction CHT } \\
\text { - } \\
\text { at the end } \\
\text { of treatment }\end{array}$ & $\begin{array}{l}\text { After adjustment } \\
\text { for } F L T 3-I T D \\
\text { status, the effect of } \\
\text { NPM1 MRD } \\
\text { appeared to be } \\
\text { similar. }\end{array}$ & No & NA & $\begin{array}{l}\text { - WT1 MRD associated with } \\
\text { increased hazard of relapse and } \\
\text { shorter OS from CR. } \\
\text { Positive NPM1-mutated MRD } \\
\text { predicted higher risk of relapse, } \\
\text { but did not influence OS } \\
\text { Achievement of negative NPM1 } \\
\text { mutation MRD significantly } \\
\text { more frequent in GO arm }\end{array}$ \\
\hline $\begin{array}{l}\text { Debarri et al., } \\
2015 \text { [50] }\end{array}$ & 31 Yes/NA & $0.1 \%$ & $\begin{array}{ll}- & \text { post } \\
& \text { induction CHT } \\
\text { - } & \text { post first and } \\
& \text { second } \\
\text { consolidation courses }\end{array}$ & $\begin{array}{l}\text { Analysis of } \\
\text { correlation } \\
\text { between NPM1 } \\
\text { mutations and } \\
I D H 1 / 2 \text { or } \\
D N M T 3 A \\
\text { mutations }\end{array}$ & $\begin{array}{l}\text { One patient with } \\
\text { IDH2 mutation } \\
\text { developed a } \\
\text { NPM1-negative } \\
\text { MDS }\end{array}$ & NA & $\begin{array}{ll}\text { - } & \text { IDH1/2 mutational status by } \\
\text { NGS predicted relapse or } \\
\text { disease evolution in } 100 \% \text { cases } \\
\text { DNMT3A mutations not } \\
\text { correlated with disease status } \\
\text { (a preleukemic clone persisted } \\
\text { in 40\% of cases in CR) }\end{array}$ \\
\hline
\end{tabular}


Table 2. Cont.

\begin{tabular}{|c|c|c|c|c|c|c|c|}
\hline Reference & $\begin{array}{c}\text { Intensive } \\
\text { CHT/HSCT } \\
\text { (No. of Patients) }\end{array}$ & $\begin{array}{l}\text { Significant MRD } \\
\text { Threshold }\end{array}$ & Prognostic Timepoints & $\begin{array}{l}\text { Correlation with } \\
\text { Other Molecular } \\
\text { Markers }\end{array}$ & Clonal Evolution & $\begin{array}{l}\text { Median Time } \\
\text { since Molecular } \\
\text { to Morphologic } \\
\text { Relapse (Range) }\end{array}$ & Clinical Relevance \\
\hline $\begin{array}{l}\text { Pettersson et al., } \\
2016 \text { [51] }\end{array}$ & 15 Yes/5 HSCT & $0.1 \%$ & During follow-up & NA & $\begin{array}{c}\text { One of } 8 \text { relapsing } \\
\text { patients developed } \\
\text { a NPM1-negative } \\
\text { relapse }\end{array}$ & NA & $\begin{array}{l}\text { RQ-PCR of NPM1 A type } \\
\text { mutation was more sensitive } \\
\text { and reliable than MFC for } \\
\text { determination of MRD }\end{array}$ \\
\hline $\begin{array}{l}\text { Karas et al., } \\
2016[52]\end{array}$ & -/60 alloHSCT & $0.1 \%$ & $\begin{array}{l}\text { pre-transplant (less than } \\
1 \text { week prior to start } \\
\text { conditioning regimen) }\end{array}$ & $\begin{array}{c}\text { FLT3-ITD } \\
\text { positivity had no } \\
\text { adverse effect on } \\
\text { HSCT outcome in } \\
\text { patients with AML } \\
\text { with NPM1 } \\
\text { mutation in CR }\end{array}$ & No & $\begin{array}{l}4 \text { months (3-13) } \\
\text { from HSCT to } \\
\text { relapse, especially } \\
\text { in patients with } \\
\text { high preHSCT } \\
\text { MRD }\end{array}$ & $\begin{array}{l}\text { - } \\
\text { older age and pre-transplant } \\
\text { MRD had independent } \\
\text { prognostic impact on EFS } \\
\text { and OS } \\
\text { 3-year relapse rate, EFS and OS } \\
\text { were } 6 \%, 72 \%, 75 \% \text { with } \\
\text { low-level MRD and } 48 \%, 35 \% \text {, } \\
40 \% \text { in patients with higher } \\
\text { MRD levels }\end{array}$ \\
\hline $\begin{array}{c}\text { Alizad } \\
\text { Ghandforoush } \\
\text { et al., 2016 [53] }\end{array}$ & $\begin{array}{l}11 \mathrm{Yes} / 6 \\
\text { alloHSCT }\end{array}$ & $<5 \log$ reduction & during follow-up & NA & No & NA & $\begin{array}{l}\text { Relapse occurred in } 6(54.5 \%) \\
\text { patients, whose NPM1 } \\
\text { mutation levels showed less } \\
\text { than } 5 \text { log reduction } \\
\text { after treatment }\end{array}$ \\
\hline $\begin{array}{l}\text { Ivey et al., } \\
2016 \text { [54] }\end{array}$ & $\begin{array}{c}346+91 \text { Yes } / 82 \\
\text { alloHSCT }\end{array}$ & $0.01 \%$ & $\begin{array}{l}\text { after second CHT cycle } \\
\text { (PB samples) }\end{array}$ & $\begin{array}{c}\text { Presence of } \\
\text { FLT3-ITD or } \\
D N M T 3 A \\
\text { mutations did not } \\
\text { provide additional } \\
\text { prognostic } \\
\text { information }\end{array}$ & $\begin{array}{l}\text { NPM1 mutations } \\
\text { detectable in } \\
69 / 70(99 \%) \\
\text { patients at relapse }\end{array}$ & $\begin{array}{l}133 \text { days }(\mathrm{BM}), 87 \\
\text { days }(\mathrm{PB})\end{array}$ & $\begin{array}{ll}\text { - } & \text { Persistence of NPM1 mutated } \\
\text { transcripts, observed in } 15 \% \text { of } \\
\text { patients after second CHT cycle, } \\
\text { associated with greater risk of } \\
\text { relapse after a 3-year follow-up } \\
\text { and lower survival rate } \\
(24 \% \text { vs. } 75 \%) \\
\text { MRD as the only independent } \\
\text { prognostic factor for death }\end{array}$ \\
\hline $\begin{array}{l}\text { Kayser et al., } \\
2016 \text { [55] }\end{array}$ & -/67 alloHSCT & $1 \%$ & prior to alloHSCT & $\begin{array}{l}\text { FLT3-ITD status } \\
\text { had no impact on } \\
\text { prognosis }\end{array}$ & No & NA & $\begin{array}{l}\text { Significant difference in OS } \\
\text { after alloHSCT between } \\
\text { pre-transplant MRD-positive } \\
\text { and MRD-negative patients } \\
\text { (estimated 5-year OS rates } \\
\text { 40\% vs. 89\%) } \\
\text { Outcome of patients with } \\
\text { preHSCT MRD positivity as } \\
\text { poor as that of cases } \\
\text { transplanted with } \\
\text { refractory disease }\end{array}$ \\
\hline
\end{tabular}


Table 2. Cont.

\begin{tabular}{|c|c|c|c|c|c|c|c|}
\hline Reference & $\begin{array}{c}\text { Intensive } \\
\text { CHT/HSCT } \\
\text { (No. of Patients) }\end{array}$ & $\begin{array}{c}\text { Significant MRD } \\
\text { Threshold }\end{array}$ & Prognostic Timepoints & $\begin{array}{l}\text { Correlation with } \\
\text { Other Molecular } \\
\text { Markers }\end{array}$ & Clonal Evolution & $\begin{array}{c}\text { Median Time } \\
\text { since Molecular } \\
\text { to Morphologic } \\
\text { Relapse (Range) }\end{array}$ & Clinical Relevance \\
\hline $\begin{array}{l}\text { Malmberg et al., } \\
2017 \text { [56] }\end{array}$ & NA/NA & $0.001 \%$ & NA & $\begin{array}{c}\text { CPS1, FAM193A, } \\
\text { ITGB7 }\end{array}$ & NA & NA & $\begin{array}{ll}\text { For mutation load of NPM1, } \\
\text { good correlation between } \\
\text { results from deep sequencing } \\
\text { and RQ-PCR } \\
\text { Targeted deep sequencing more } \\
\text { sensitive for MRD } \\
\text { quantification than MFC }\end{array}$ \\
\hline $\begin{array}{l}\text { Balsat et al., } \\
2017 \text { [57] }\end{array}$ & $\begin{array}{c}152 \text { Yes } / 44 \\
\text { alloHSCT }\end{array}$ & $\begin{array}{c}\text { 4-log reduction in } \mathrm{PB} \\
\mathrm{MRD}\end{array}$ & $\begin{array}{l}\text { post-induction CHT } \\
\text { cycle }\end{array}$ & $\begin{array}{l}\text { Abnormal } \\
\text { karyotype, } \\
\text { FLT3-ITD, PB } \\
\text { MRD associated } \\
\text { with higher } \\
\text { relapse incidence } \\
\text { and shorter OS. }\end{array}$ & No & NA & $\begin{array}{ll}\text { - } & \text { Patients without early MRD } \\
\text { reduction had higher incidence } \\
\text { of relapse and shorter OS } \\
\text { - In patients with non favorable } \\
\text { AML, HSCT improved } \\
\text { outcomes only in case of }<4 \text {-log } \\
\text { reduction PB-MRD }\end{array}$ \\
\hline $\begin{array}{l}\text { Schieppati et al., } \\
2017 \text { [58] }\end{array}$ & $\begin{array}{l}68 \text { Yes/9 } \\
\text { alloHSCT }\end{array}$ & $0.5 \%$ & TP1 (BM), TP3 (PB) & $\begin{array}{l}\text { FLT3-ITD did not } \\
\text { impact on } \\
\text { relapse risk }\end{array}$ & No & NA & $\begin{array}{l}\text { Molecular MRD monitoring of } \\
\text { crucial importance in detecting } \\
\text { relapse at an early stage }\end{array}$ \\
\hline $\begin{array}{l}\text { Mencia-Trinchant } \\
\text { et al., } 2017 \text { [59] }\end{array}$ & 3 Yes/- & NA & NA & NA & No & NA & $\begin{array}{l}\text { Novel ddPCR technique } \\
\text { composed of massively } \\
\text { multiplex pools of } \\
\text { insertion-specific primers that } \\
\text { selectively detected virtually al } \\
\text { NPM1 mutations in a manner } \\
\text { that was robust to clonal } \\
\text { heterogeneity and did not } \\
\text { require NPM1 } \\
\text { sequence information }\end{array}$ \\
\hline $\begin{array}{l}\text { Getta et al., } \\
2017 \text { [60] }\end{array}$ & -/104 alloHSCT & $<5 \% \mathrm{VAF}$ & before HSCT & NA & No & NA & $\begin{array}{l}\text { - Mutations in DNMT3A, TET2, } \\
\text { JAK2 less likely to be cleared } \\
\text { than NPM1 (negative in } 8 / 9 \\
\text { cases), IDH1/2, FLT3-ITD } \\
\text { MRD detected concurrently } \\
\text { with MFC and NGS conferred } \\
\text { the highest relapse risk }\end{array}$ \\
\hline
\end{tabular}


Table 2. Cont.

\begin{tabular}{|c|c|c|c|c|c|c|c|}
\hline Reference & $\begin{array}{c}\text { Intensive } \\
\text { CHT/HSCT } \\
\text { (No. of Patients) }\end{array}$ & $\begin{array}{l}\text { Significant MRD } \\
\text { Threshold }\end{array}$ & Prognostic Timepoints & $\begin{array}{l}\text { Correlation with } \\
\text { Other Molecular } \\
\text { Markers }\end{array}$ & Clonal Evolution & $\begin{array}{c}\text { Median Time } \\
\text { since Molecular } \\
\text { to Morphologic } \\
\text { Relapse (Range) }\end{array}$ & Clinical Relevance \\
\hline $\begin{array}{l}\text { Bill et al., } \\
2018 \text { [61] }\end{array}$ & $51 \mathrm{Yes} / 51 \mathrm{HSCT}$ & $0.01 \%$ & prior to HSCT & $\begin{array}{l}\text { Adverse } \\
\text { prognostic role of } \\
\text { NPM1 mutation } \\
\text { MRD independent } \\
\text { of other known } \\
\text { prognostic } \\
\text { markers }\end{array}$ & NA & $\begin{array}{l}101 \text { days after } \\
\text { HSCT (median } \\
\text { time to relapse for } \\
\text { all patient cohort) }\end{array}$ & $\begin{array}{l}\text { - } \\
\text { MRD positive before HSCT } \\
\text { - } \quad \text { The 2-year cumulative } \\
\text { incidence of relapse was } 64.7 \% \\
\text { vs. } 6 \% \text { translating into OS } \\
38.8 \% \text { vs. } 71.7 \% \text { in pre HSCT } \\
\text { MRD+ and } \\
\text { MRD-negative, respectively }\end{array}$ \\
\hline $\begin{array}{l}\text { Jongen-Lavrencic } \\
\text { et al., } 2018 \text { [62] }\end{array}$ & 430 (168) Yes / & $2.5 \%$ allele frequency & $\begin{array}{l}\text { samples obtained } \\
\text { during a defined period } \\
\text { of remission, between } \\
21 \text { days and } 4 \text { months } \\
\text { after start of second } \\
\text { treatment cycle }\end{array}$ & - & NA & NA & $\begin{array}{ll}\text { - } & \text { mutations persisted in } 51.4 \% \text { of } \\
\text { patients during CR } \\
\text { - } \\
\text { Persistent DTA mutations not } \\
\text { correlated with increased } \\
\text { relapse rate } \\
\text { - Persistent non-DTA mutations } \\
\text { conferred higher relapse rates } \\
\text { and lower survival rates }\end{array}$ \\
\hline $\begin{array}{l}\text { Zhou et al., } \\
2018 \text { [63] }\end{array}$ & -/59 alloHSCT & $0.01 \%$ & $\begin{array}{l}\text { pre-HSCT and post } \\
\text { HSCT (around day }+28 \text { ) }\end{array}$ & $\begin{array}{l}\text { Peri-HSCT MRD } \\
\text { independent } \\
\text { prognostic factor }\end{array}$ & No & NA & $\begin{array}{l}\text { - } \quad \text { Before HSCT MRD detected by } \\
\text { MFC was the most significant } \\
\text { risk factor for relapse } \\
\text { NGS testing of NPM1-mutated } \\
\text { post-HSCT improved the risk } \\
\text { assessment of relapse }\end{array}$ \\
\hline $\begin{array}{l}\text { Zappasodi et al., } \\
2018 \text { [64] }\end{array}$ & $\begin{array}{l}201 \mathrm{Yes} / 4 \\
\text { alloHSCT }\end{array}$ & NA & $\begin{array}{l}\text { MRD negativity at any } \\
\text { time, during or after the } \\
\text { end of 1st line treatment }\end{array}$ & NA & No & NA & $\begin{array}{ll}- & \text { molecular CR obtained in } \\
\text { 73.7\% of NPM1-mutated cases } \\
\text { molecular CR at the end of } \\
\text { treatment prognostic factor for } \\
\text { DFS and OS in } \\
\text { NPM1-mutated AML }\end{array}$ \\
\hline $\begin{array}{c}\text { Delsing } \\
\text { Malmberg et al., } \\
2018 \text { [65] }\end{array}$ & $-/ 29$ alloHSCT & $0.02 \%$ & pre and post-HSCT & $\begin{array}{c}\text { MRD was an } \\
\text { independent risk } \\
\text { factor associated } \\
\text { with clinical } \\
\text { outcome }\end{array}$ & No & $\begin{array}{c}4.5 \text { months (3.5-11) } \\
\text { for MRD+; } 7.7 \\
\text { months (7-32) for } \\
\text { MRD-cases }\end{array}$ & $\begin{array}{l}\text { Post-HSCT deep sequencing } \\
\text { MRD status was significantly } \\
\text { associated with clinical } \\
\text { outcome (3-year OS 20\% } \\
\text { vs. } 89 \% \text { ) }\end{array}$ \\
\hline
\end{tabular}


Table 2. Cont

\begin{tabular}{|c|c|c|c|c|c|c|c|}
\hline Reference & $\begin{array}{c}\text { Intensive } \\
\text { CHT/HSCT } \\
\text { (No. of Patients) }\end{array}$ & $\begin{array}{l}\text { Significant MRD } \\
\text { Threshold }\end{array}$ & Prognostic Timepoints & $\begin{array}{c}\text { Correlation with } \\
\text { Other Molecular } \\
\text { Markers }\end{array}$ & Clonal Evolution & $\begin{array}{c}\text { Median Time } \\
\text { since Molecular } \\
\text { to Morphologic } \\
\text { Relapse (Range) }\end{array}$ & Clinical Relevance \\
\hline $\begin{array}{l}\text { Kapp-Schwoerer } \\
\text { et al., } 2018 \text { [66] }\end{array}$ & $\begin{array}{l}611 \text { Yes } / 162 \\
\text { alloHSCT }\end{array}$ & $2 \%$ & $\begin{array}{c}\text { post two CHT cycles } \\
\text { (BM) }\end{array}$ & $\begin{array}{l}\text { NPM1 MRD } \\
\text { negativity } \\
\text { associated with } \\
\text { lower relapse rate } \\
\text { and better OS } \\
\text { independent of } \\
\text { DNMT3A and } \\
\text { FLT3-ITD } \\
\text { mutations }\end{array}$ & No & NA & 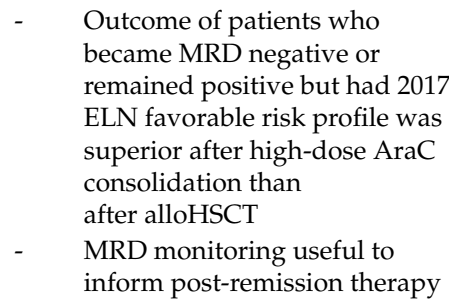 \\
\hline $\begin{array}{l}\text { Caprioli et al., } \\
2018 \text { [67] }\end{array}$ & $-/ 27$ alloHSCT & $0.01 \%$ & pre-alloHSCT & NA & No & NA & $\begin{array}{ll}\text { - } & \text { molecular analysis had higher } \\
\text { sensitivity than MFC } \\
\text { - } \quad \text { Low-level or negative MRD } \\
\text { associated with improved LFS }\end{array}$ \\
\hline $\begin{array}{l}\text { Patkar et al., } \\
2018 \text { [68] }\end{array}$ & 83 Yes/- & $\begin{array}{l}\text { 1-log cut-off between post } \\
\text { induction and post } \\
\text { consolidation }\end{array}$ & $\begin{array}{l}\text { post induction and post } \\
\text { consolidation }\end{array}$ & $\begin{array}{l}\text { NGS-MRD for } \\
\text { NPM1 mutations } \\
\text { as the most } \\
\text { independent } \\
\text { prognostic factor }\end{array}$ & No & NA & $\begin{array}{l}\text { - } \\
\text { NGS was an useful test for } \\
\text { prediction of relapse } \\
\text { and survival } \\
\text { NGS and MFC may be } \\
\text { complementary, with patients } \\
\text { MRD-negative by both } \\
\text { techniques having } \\
\text { excellent outcome }\end{array}$ \\
\hline $\begin{array}{l}\text { Onecha et al., } \\
2018 \text { [69] }\end{array}$ & $\begin{array}{l}50 \text { Yes } / 7 \\
\text { alloHSCT }\end{array}$ & $\begin{array}{c}0.1 \% \text { post } \\
\text { induction } / 0.025 \% \text { post } \\
\text { consolidation }\end{array}$ & $\begin{array}{l}\text { post induction and post } \\
\text { consolidation }\end{array}$ & $\begin{array}{l}\text { Higher risk of } \\
\text { death in subjects } \\
\text { with advanced age, } \\
\text { MRD+ status or } \\
\text { with FLT3-ITD }\end{array}$ & No & NA & $\begin{array}{l}\text { MRD+ status post induction } \\
\text { and post consolidation } \\
\text { associated with lower OS and } \\
\text { shorter DFS and OS ( } 33 \% \text { vs } \\
81 \% \text { ), respectively } \\
\text { NGS improved the capacity to } \\
\text { predict AML outcome over } \\
\text { MFC or RQ-PCR }\end{array}$ \\
\hline
\end{tabular}


Table 2. Cont.

\begin{tabular}{|c|c|c|c|c|c|c|c|}
\hline Reference & $\begin{array}{c}\text { Intensive } \\
\text { CHT/HSCT } \\
\text { (No. of Patients) }\end{array}$ & $\begin{array}{c}\text { Significant MRD } \\
\text { Threshold }\end{array}$ & Prognostic Timepoints & $\begin{array}{l}\text { Correlation with } \\
\text { Other Molecular } \\
\text { Markers }\end{array}$ & Clonal Evolution & $\begin{array}{l}\text { Median Time } \\
\text { since Molecular } \\
\text { to Morphologic } \\
\text { Relapse (Range) }\end{array}$ & Clinical Relevance \\
\hline $\begin{array}{l}\text { Petrova et al., } \\
2018 \text { [70] }\end{array}$ & $22 \mathrm{Yes} / \mathrm{NA}$ & NA & NA & $\begin{array}{l}\text { NPM1 mutation } \\
\text { MRD more } \\
\text { sensitive than } \\
\text { IDH1/2-based } \\
\text { MRD }\end{array}$ & NA & NA & $\begin{array}{l}\text { - } \quad \text { ddPCR more sensitive than } \\
\text { NGS for IDH1/2 MRD detection } \\
\text { In the absence of more sensitive } \\
\text { markers, IDH1/2 mutations can } \\
\text { be used as reliable markers for } \\
\text { MRD monitoring }\end{array}$ \\
\hline $\begin{array}{l}\text { Prata et al., } \\
2018[71]\end{array}$ & $\begin{array}{c}0 / 0 \\
\text { (34 patients } \\
\text { received upfront } \\
\text { therapy with } \\
\text { HMA) }\end{array}$ & NA & $\begin{array}{l}>3 \log N P M 1 \text { MRD } \\
\text { reduction observed in } \\
4 / 6 \text { patients after } \\
6 \text { cycles, but three of } \\
\text { them relapsed within } \\
<6 \text { months of this } \\
\text { reduction. }\end{array}$ & $\begin{array}{l}\text { No factors, } \\
\text { including type of } \\
\text { HMA, FLT3 and } \\
\text { IDH1/2 status, } \\
\text { were prognostic of } \\
\text { response or } \\
\text { survival }\end{array}$ & NA & NA & $\begin{array}{ll}\text { - } & \text { Overall response rate } 45 \% \text { (CR } \\
\text { in 23.5\% of patients) } \\
\text { - } \quad \text { Median OS } 280 \text { days } \\
\text { - } & \text { No difference in OS between } \\
\text { cohorts with or without NPM1 } \\
\text { mutations suggesting limited } \\
\text { therapeutic impact of HMA in } \\
\text { NPM1-mutated AML }\end{array}$ \\
\hline $\begin{array}{l}\text { Ottone et al., } \\
2018 \text { [72] }\end{array}$ & 177 Yes/NA & NA & NA & $\begin{array}{l}\text { Evaluation of } \\
\text { correlation with } \\
D N M T 3 A \text { and } \\
\text { FLT3 mutations }\end{array}$ & NA & NA & $\begin{array}{l}\text { NPM1-mutated transcripts } \\
\text { levels correlated with disease } \\
\text { course, as a reliable } \\
\text { MRD marker } \\
\text { DNMT3A } A^{\mathrm{R} 882 \mathrm{H}} \text { levels did not } \\
\text { reflect AML status }\end{array}$ \\
\hline $\begin{array}{l}\text { Gaksch et al., } \\
2018 \text { [73] }\end{array}$ & 34 Yes/- & $\mathrm{VAF}<0.5 \%$ & $\begin{array}{l}\text { After at least one } \\
\text { consolidation cycle }\end{array}$ & $\begin{array}{l}\text { In multivariate } \\
\text { analysis including } \\
\text { age, leukocyte } \\
\text { count and genetic } \\
\text { risk, residual } \\
\text { disease positivity } \\
\text { remained } \\
\text { statistically } \\
\text { significant as an } \\
\text { adverse factor } \\
\text { for RFS }\end{array}$ & NA & NA & $\begin{array}{l}\text { Persistence of non-DNMT3A } \\
\text { mutations was significantly } \\
\text { associated with higher risk of } \\
\text { AML relapse and shorter RFS, } \\
\text { with a trend for shorter OS. } \\
\text { Strong concordance between } \\
\text { NGS and ddPCR for } \\
\text { NPM1 mutations }\end{array}$ \\
\hline
\end{tabular}

MRD, minimal/measurable residual disease; AML, acute myeloid leukemia; CHT, chemotherapy; HSCT, hematopoietic stem cell transplantation; NA; not available; alloHSCT, allogeneic HSCT; RFS, relapse-free survival; OS, overall survival; CR, complete remission; EFS, event-free survival; BM, bone marrow; PB, peripheral blood; NCN, normalized copy number; NGS, next-generation sequencing; RQ-PCR, real-time quantitative polymerase chain reaction; DFS, disease-free survival; ELN, European Leukemia Net; GO, gemtuzumab ozogamycin; MFC, multiparametric flow cytometry; TP, timepoint; ddPCR, digital droplet PCR; DTA (DNMT3A, TET2, ASXL1); VAF, variant allele frequency; LFS, leukemia-free survival, HMA, hypomethylating agents (azacitidine, decitabine, guadecitabine). 


\section{Prognostic MRD Thresholds and Relevant Timepoints for NPM1 Mutations}

As above mentioned, several clinical trials have so far validated the clinical relevance of molecular MRD monitoring in NPM1-mutated AML patients (Table 2), trying to identify prognostic MRD thresholds and most relevant timepoints for collecting either BM or PB samples [30-73]. Schnittger et al. retrospectively analyzed 1227 diagnostic and follow-up samples in 252 NPM1-mutated AML patients by RQ-PCR assays [35]. A total of 47 relapses were predictable due to NPM1 mutation levels increase of at least $1 \log$ or in 15 cases because of a less than 3-log reduction in NPM1 mutation levels. High prognostic value for MRD monitoring was documented at four different intervals after therapy initiation (namely 18-60 days, 61-120 days, 121-365 days, $>365$ days), with levels of residual NPM1 transcripts as the most relevant factor affecting event-free survival (EFS) in multivariate analysis, also in the subset of patients undergoing hematopoietic stem cell transplantation (HSCT). Moreover, MRD thresholds of $0.01 \%$ and $0.1 \%$ NPM1-mutated / ABL1 obtained during first-line treatment and after HSCT or second-line therapy, respectively, discriminated between prognostic subgroups, but without influencing overall survival (OS) [35]. Another German-Austrian group evaluated MRD prognostic role in 245 intensively treated adults with NPM1-mutated AML [40]. Achievement of RQ-PCR negativity after double induction therapy identified patients with low 4-year cumulative incidence of relapse (CIR), namely $6.5 \%$ compared with $53 \%$, for subjects with persisting MRD positivity. This also translated into significant differences in OS (90\% versus 51\%, respectively; $p=0.001)$. Furthermore, according to Kronke et al., NPM1-mutated transcript levels were an independent prognostic factor also when analyzed after completion of consolidation therapy (4-year OS $80 \%$ and $44 \%$ for MRD-negative and MRD-positive patients, respectively) and during follow-up, when serial MRD monitoring allowed early prediction of AML relapse in patients exceeding an arbitrary cut-off value of greater than $2 \%$ NPM1-mutated/ABL1 [40]. Shayegi et al. subsequently investigated the prognostic impact of different NPM1-mutated MRD cut-off values, among 155 AML patients treated within intensive treatment protocols [44]. They found that MRD levels $>1 \%$ after completion of conventional chemotherapy were associated with increased relapse risk and significantly worse survival outcomes, whereas for patients tested after having undergone HSCT, the best prognostic cut-off value for disease-free survival (DFS) and OS was 10\% NPM1-mutated / ABL1 [44]. Interestingly, Hubmann et al. monitored molecular MRD during aplasia, after induction and consolidation chemotherapy, and during follow-up in 158 patients with NPM1-mutated AML [47]. The assessment of MRD, with a cut-off ratio of 0.01 for absolute NPM1-mutated ratios and a 3-log relative reduction, after induction therapy resulted the most appropriate checkpoint in order to identify patients in CR at high risk of relapse. Indeed, the authors observed 2-year CIR 77.8\% and 26.4\% for MRD-positive and MRD-negative after induction, respectively. In this series, early molecular response thus seemed to be more prognostically relevant than persistent MRD positivity after consolidation treatments [47]. Subsequently, Ivey et al. reported serial prospective NPM1 mutation MRD monitoring in 346 AML patients enrolled in NCRI AML17 trial [54]. The major finding of this large clinical study was that persistence of NPM1-mutated transcripts in PB samples exceeding a value of $0.01 \%$ NPM1-mutated/ABL1 after the second chemotherapy cycle was associated with a greater risk of relapse at a 3-year follow-up than was the absence of such mutated transcripts $(82 \%$ versus $30 \%$, respectively; $p<0.001)$. Notably, MRD positivity after two treatment cycles was observed in $15 \%$ of patients and also translated into lower OS rates in these subjects compared with MRD-negative patients ( $24 \%$ versus $75 \%$, respectively; $p<0.001)$. Additionally, the presence of MRD at this testing timepoint was the only independent prognostic factor for death in multivariate analysis and superseded other adverse baseline cytogenetic or molecular factors, such as FLT3-ITD or DNMT3A mutations [54]. More recently, Balsat et al. reported on MRD evaluation in 152 NPM1-mutated AML patients, showing that patients who did not achieve a 4-log reduction in NPM1-mutated transcripts in PB samples after induction therapy ( $45 \%$ of study cohort) had a higher 3-year CIR (65.8\% versus $20.5 \%)$ and a shorter 3-year OS (40.8\% versus $91.2 \%$ ) compared with patients who obtained an adequate MRD reduction [57]. Interestingly, the outcome of patients with a positive PB or BM MRD but with $>4$-log reduction in PB MRD after induction treatment had a similar risk of relapse to subjects with 
both MRD negativity and $>4$-log reduction in PB MRD. Notably, in the subgroup of patients with FLT3-ITD, only age, white blood cell (WBC) count and <4-log reduction in PB NPM1-mutated MRD after induction, but not FLT3-ITD allelic ratio, were identified as prognostic factors. Among the 71 patients with non-favorable risk AML according to ELN risk stratification, DFS and OS were significantly improved by allogeneic HSCT only in those with a $<4$-log reduction in PB MRD, whereas this benefit was not documented in those with an MRD clearance $>4-\log$, thereby suggesting the potential use of early MRD investigation in PB as a predictive factor for HSCT indication [57].

Collectively, the results of the studies presented above confirmed that RQ-PCR is a reliable molecular tool to assess MRD in most patients with NPM1-mutated AML [35,40,44,47,54,57]. The MRD monitoring, by either a kinetic measure $[32,35,47,53,57,68]$ or the obtainment of an absolute threshold of NPM1-mutated transcripts $[31,33,35,38,40,44,47-52,54-56,58,61,63,65-67,69]$, was overall predictive of relapse and survival outcomes (Table 2). However, due to differences in patients' cohorts and the lack of standardization of NPM1-mutated RQ-PCR MRD assays and analytical methods, any comparison between different studies remains extremely difficult, and evidence-based conclusions on the best MRD thresholds and timepoints cannot be definitely drawn $[8,44,75]$. Interestingly, in the study by Balsat et al., a positive MRD determination in BM and concurrent negativity in PB samples was found in $24.6 \%$ of cases [57], in concordance with the $14.5 \%$ reported by Shayegi et al. in MRD samples collected at different timepoints [44]. Absolute differences in NPM1-mutated RQ-PCR sensitivity generally favor $\mathrm{BM}$ over $\mathrm{PB}$ on the order of $0.6-1.5 \log _{10}[44,54,57,75]$, so that $\mathrm{BM}$ aspirate was initially considered to be the best source to investigate MRD [75]. Also, in the series by Ivey et al., serial monitoring of $\mathrm{PB}$ and $\mathrm{BM}$ paired samples confirmed that the BM analysis increased the rate of detection of MRD, affording a median $1-\log _{10}$ increment in sensitivity [54]. However, the presence of NPM1-mutated transcripts, with a $0.01 \%$ absolute threshold to define positivity, in PB of patients after the second chemotherapy cycle was highly discriminatory and was the only significant prognostic factor [54]. It has thus been hypothesized that MRD assessment in BM could overestimate the quality of response due to the risk of BM samples dilution by $\mathrm{PB}$, thereby justifying PB MRD monitoring at least at definite timepoints $[2,44,54,75]$, perhaps with $0.01 \%$ cut-off value to define MRD negativity, even though using a $>4$-log reduction could also be considered reasonable [75]. Monitoring MRD in PB samples also offers other advantages, such as decreased procedural morbidity and the possibility of more frequent testing [8].

\section{NPM1 Mutation Levels at AML Diagnosis}

In most series, NPM1-mutated transcript levels at diagnosis did not correlate with the type of NPM1 mutation or with other clinical characteristics, namely age, sex, WBC count, BM blast count, lactate dehydrogenase level, ELN risk stratification or FLT3 gene mutational status [7,9,12,19,26]. On the contrary, Balsat et al. found higher median NPM1-mutated baseline levels in cases with WBC count greater than $50 \times 10^{9} / \mathrm{L}$ or with FLT3-ITD positivity, whereas no significant correlation was documented with age, karyotype or other concurrent gene mutations [29]. However, it should be noted that pretreatment NPM1-mutated expression levels detected by RQ-PCR did not influence CR rate after induction chemotherapy [19], CIR [19] and survival parameters, such as EFS and OS $[7,12,19,26]$. Conversely, Patel et al. recently reported targeted sequencing data from 109 patients with NPM1-mutated AML to retrospectively evaluate the potential significance of NPM1 variant allele frequency (VAF) at diagnosis, comutations and clinical features on patient outcomes [76]. Interestingly, high NPM1 VAF $(\geq 0.44)$ observed on NGS correlated with shortened median OS (12.1 months versus not reached, $p<0.0001$ ) as well as median EFS (7.5 versus 65.4 months, $p<0.0001$ ). High NPM1-mutated allele burden at diagnosis was an independent predictor of unfavorable clinical outcomes, particularly in patients who received HSCT in first CR and in the subgroup of subjects with concomitant DNMT3A mutations. Because of BM multilineage involvement of NPM1 mutations not being restricted to cells with blast morphology $[12,16,20]$, the VAF could in fact reveal the true clonal disease burden. Higher NPM1 mutant allele burden may be less amenable to eradication by 
intensive chemotherapy, potentially resulting in a higher likelihood of MRD persistence. Alternatively, high NPM1-mutated allele burden may indicate the presence of disease in different hematopoietic cell populations, belonging to the NPM1-mutated leukemic clone regardless of blast morphology, which could be intrinsically resistant to chemotherapy and could subsequently foster leukemia relapse, resulting in adverse clinical outcomes [76].

\section{Sequential MRD Monitoring and Molecular Prediction of Relapse}

Several studies have investigated whether NPM1-mutated MRD monitoring during follow-up of AML patients in CR after the end of intensive treatments could foresee hematologic relapse [31,33-35,37-40,44,47,52,54,61,65]. Notably, post-treatment serial testing may portend morphologic recurrence either when, in previously MRD-negative patients, NPM1-mutated transcripts again become detectable or, alternatively, when rising MRD levels are observed in patients with persistent low-level MRD positivity [75]. Ommen et al. documented that kinetics of molecular relapse was markedly different among AML with NPM1 mutations or with rearrangements such as PML-RARA, RUNX1-RUNX1T1 or CBFb-MYH11, and subsequently developed a mathematical model to predict the time elapsing between molecular and hematologic relapse [38]. According to this model, AML positive for $C B F b-M Y H 11$ displayed a slower clone regrowth than AML with other molecular signatures, including NPM1 mutations [38,77]. In many patients who previously obtained molecular CR, reoccurrence of NPM1-mutated transcripts may be detectable by molecular techniques over 2-3 months before overt morphologic relapse occurred [1,31,33-35,37-40,44,47,52,54,61,65], as summarized in Table 2, with significant differences observed between patients with concurrent FLT3-ITD positivity or negativity (65 versus 120 days, respectively) [38]. Furthermore, median time since molecular to hematologic relapse could also have a broad range, as documented by Kronke et al. (median 2.6 months, range 0.4 to 23.6) [40], and may significantly vary according to the NPM1-mutated/ABL1 cut-off value considered for MRD positivity, as shown in the study Shayegi et al. [44]. Finally, Ivey et al. observed a longer time to overt relapse if molecular MRD was first detected in BM rather than in PB samples (133 versus 87 days, respectively) [54]. These data collectively suggest that serial molecular MRD assessment during follow-up may provide a timely prediction of impending morphologic relapse in NPM1-mutated AML patients, potentially allowing pre-emptive therapeutic approaches, preferably in the context of clinical trials $[1,75,77]$.

\section{MRD Levels and Allogeneic Hematopoietic Stem Cell Transplant in NPM1-Mutated AML}

The use of alloHSCT in patients with NPM1-mutated AML is still controversial. In the retrospective study by Rollig et al., a significant improvement in RFS was documented in the donor group, indicating a beneficial effect of alloHSCT in NPM1-mutated AML patients in first CR, also observed in the favorable subgroup without FLT3-ITD [78]. Even in the absence of a significant difference in OS, most likely as a result of the fact that NPM1-mutated AML patients experiencing relapse often achieved response to salvage treatments, alloHSCT could represent a valuable consolidation option for NPM1-mutated AML patients in first CR if a sibling donor is available, especially in young patients, with a relatively low risk of non-relapse mortality [78]. Unfortunately, prospective MRD monitoring was not performed within this trial [78]. Notably, several subsequent studies investigated the prognostic significance of pre-transplant NPM1-mutated MRD levels (Table 2) $[52,55,57,60,61,63,65,67]$. In detail, Karas et al., among 60 NPM1-mutated AML patients undergoing alloHSCT, identified a significantly negative prognostic impact on EFS and OS, only for age above 63 years and for pre-transplant MRD positivity, immediately before starting the conditioning regimen, with cut-off $0.1 \%$ [52]. The estimated probabilities of 3-year relapse, EFS and OS were $6 \%, 72 \%$ and $75 \%$, compared with $48 \%, 35 \%$ and $40 \%$ for patients with lower and higher levels of MRD, respectively [52]. Kayser et al. reported on 67 NPM1-mutated AML patients, receiving alloHSCT in either first (31 cases) or second (20 cases) CR or with refractory disease (16 cases) [55]. Overall, patients transplanted in CR had significantly longer OS as compared to patients with 
refractory disease. However, for patients undergoing alloHSCT in CR, there was a highly significant difference in OS between pre-transplant MRD-negative and MRD-positive cases, with estimated 5 -year OS rates of $89 \%$ versus $40 \%$, respectively. Interestingly, these latter patients had an OS rate comparable to that observed in patients receiving alloHSCT with refractory disease (38\%). According to Kayser et al., pre-transplant NPM1-mutated MRD positivity $(>1 \%)$ was a significant predictor of poor outcome after alloHSCT, independently of other variables, such as FLT3-ITD mutational status, BM blast count at diagnosis and age [55]. More recently, Bill et al. applied highly sensitive ddPCR to quantify pre-transplant NPM1-mutated MRD in 51 patients [61]. The 2-year CIR was $64.7 \%$ versus $6 \%$, translating into OS rate $38.8 \%$ versus $71.7 \%$ in pre-transplant MRD-positive and MRD-negative cases, respectively. Thus, pre-transplant MRD persistence independently predicted adverse outcomes also in this cohort, including a large proportion of older patients $(86.3 \%$ of cases, with median age 62 years), mainly receiving non-myeloablative conditioning regimens [61]. Interestingly, the recommendation to perform alloHSCT only based on other known prognostic factors, without considering pre-transplant NPM1-mutated MRD levels, is currently questionable $[52,55,61]$. According to Balsat et al., pre-transplant MRD assessment demonstrated that a survival benefit from alloHSCT was not seen for patients with $>4-\log$ NPM1-mutated MRD clearance, whereas it was maintained for those with insufficient MRD clearance, suggesting the relevant use of MRD evaluation in transplant selection, to be further validated in clinical studies $[57,75]$. However, it is so far uncertain whether patients with persistent MRD positivity could benefit from additional chemotherapy cycles before alloHSCT or, alternatively, from intensification of conditioning regimen, whenever possible. Prospective clinical trials are warranted to identify treatment options to improve the prognosis of NPM1-mutated AML patients resulting MRD-positive prior to alloHSCT [61].

Furthermore, since the first observation in a small patient cohort by Bacher et al. [34], post-transplant NPM1-mutated MRD monitoring has been shown to be predictive of relapse in several large retrospective series $[35,36,44,63,65]$. Schnittger et al. reported a significant effect on survival of NPM1 mutation levels, with relevant threshold $0.1 \%$, for samples analyzed between days 61 and 120 and between 121 and 365 after alloHSCT [35]. Similarly, Shayegi et al. documented that an increasing MRD burden exceeding the threshold of 10\% NPM1-mutated / ABL1 on RQ-PCR assay was strongly associated with an increased risk of relapse, therefore resulting in significantly worse outcomes in terms of DFS and OS [44]. Most recently, Zhou et al. retrospectively investigated the application of NGS to improve prediction of post-alloHSCT relapse in 59 adults with NPM1-mutated AML [63]. While pre-transplant MFC testing identified a subset of high-risk patients in whom additional therapy before HSCT could potentially be useful; high-sensitivity NGS analysis of NPM1-mutated around day 28 after alloHSCT predicted morphologic relapse in 15 of 18 cases $(83 \%)$. Peri-transplant MRD evaluation, improving the risk assessment for relapse, may identify high-risk patients who could benefit from pre-emptive treatments after alloHSCT [63]. Moreover, Delsing Malmberg et al. reported that MRD analysis using targeted deep sequencing 3 months after alloHSCT was a strong independent predictor of both relapse risk and OS in adult NPM1-mutated AML patients [65]. Specifically, the 3-year OS was $20 \% \pm 17.9 \%$ versus $88.6 \% \pm 7.8 \%(p<0.001)$ for MRD-positive and MRD-negative patients, respectively [65]. The above-presented data clearly suggest that NPM1-mutated AML patients undergoing alloHSCT should be carefully monitored by MRD assays after transplant in order to early detect imminent morphologic relapse $[55,61]$. However, the use of early pre-emptive strategies, namely tapering of immunosuppression, donor lymphocyte infusions, administration of hypomethylating agents or other targeted drugs, in patients with persisting or re-occurring NPM1-mutated MRD positivity after alloHSCT, are not currently standardized $[55,61,75,79]$. Based upon the pivotal RELAZA phase 2 study, which evaluated the role of 5-azacitidine in 20 either AML or myelodysplastic syndrome (MDS) patients with MRD positivity after alloHSCT [80], Platzbecker et al. recently reported the results of RELAZA2 trial, which investigated MRD monitoring in 205 patients with either advanced MDS (27 cases) or AML (178 cases, including 31 with NPM1 mutations), in CR after either conventional chemotherapy only (58 cases) or consecutive alloHSCT (147 cases) [81]. 
In 53 patients who became MRD-positive at a median of 100 days after start of screening, pre-emptive 5 -azacitidine was administered, resulting in overall response rates of $71 \%$ and $48 \%$ in patients with and without antecedent alloHSCT, respectively. At a median follow-up of 13 months, OS rate was $76 \%$, suggesting that MRD-guided therapy with an hypomethylating agent may prevent or substantially delay morphologic relapse in high risk MDS or AML patients [81]. Moreover, Sockel et al. pre-emptively treated with 5-azacitidine 10 NPM1-mutated AML patients in first or second CR after intensive chemotherapy or autologous or allogeneic HSCT (3 cases), who experienced either molecular relapse or persistently detectable MRD positivity in sequential RQ-PCR analyses [82]. Molecular response, with at least 1-log decrease in the MRD level compared with pretreatment value, was obtained in 7 of the 10 patients. At a median follow-up of 10 months (range 2-12), a median of 5 cycles of 5 -azacitidine were administered and the 7 patients remained in morphologic $C R$, suggesting a potential efficacy of 5-azaciditine in controlling NPM1-mutated MRD [82]. Although post-transplant early cellular or pharmacologic preemptive strategies to enhance graft-versus-leukemia effect or to eradicate persistent MRD have preliminarily demonstrated improved outcomes, their definitive clinical efficacy should be further investigated and determined in randomized clinical trials [79].

\section{MRD Assessment in Elderly Patients with NPM1-Mutated AML}

Incidence of AML is highest among older subjects, with a median age at disease onset of 67 years $[83,84]$. Despite the use of intensive chemotherapy in fit elderly patients, clinical outcomes remain generally dismal, with lower $\mathrm{CR}$ rates (around $40-50 \%$ ) and few long-term survivors compared with younger patients $[85,86]$. Indeed, even when short-term benefits have been demonstrated, very few patients older than 60 years actually become long-term survivors, with OS rates reported to be $<10 \%$ at 3 years and $<5 \%$ at 5 years $[85,86]$. In addition to comorbidities, which commonly hamper intensive treatment approaches, it should be noted that the genetic profile of AML in the elderly is often unfavorable, with a high incidence of adverse-risk karyotypes and a lower frequency of good-risk molecular features [83]. Accordingly, NPM1 mutations are found in only 20-25\% of older AML patients, and in $35-40 \%$ of those with normal karyotype $[83,87]$. The possibility of obtaining CR remains relatively high $(60-80 \%)$ in elderly NPM1-mutated AML patients $[83,88]$. However, while NPM1-mutated AML in younger adults is generally associated with favorable clinical outcomes, especially in the absence of FLT3-ITD, in older patients the risk of relapse is markedly higher (1-year CIR $71 \%$ in patients aged $>65$ years) and survival significantly lower (2-year OS 19\%) [88]. In this disappointing clinical setting, NPM1-mutated MRD monitoring may potentially be useful for patients who obtain morphologic $C R$ in order to define the best personalized post-remissional treatment approach and to early detect relapse [83]. However, although multiple series evaluating NPM1-mutated MRD also enrolled some patients aged $>60$ years (Table 1 ) $[31-37,39,42-45,47-56,58,60-63,65,67,69,71]$, no study so far specifically focused on application and clinical significance of MRD monitoring in elderly patients with NPM1-mutated AML. Since the most predictive timepoints and MRD thresholds appear to be highly variable even between cohorts of younger adult patients, they cannot be easily applied to elderly subjects with poor prognosis and future studies are needed to further address these underexplored issues $[83,89]$. However, older AML patients frequently receive low to moderate-intensity treatments, mainly with hypomethylating agents, able to prolong survival even without the achievement of morphologic $\mathrm{CR}$, thereby raising further controversies about the relevance of MRD studies in this clinical context $[71,83]$.

\section{Clonal Evolution and Loss of NPM1 Mutation at AML Relapse}

As expected for founder genetic lesions, NPM1 gene mutation is generally stable throughout the course of the disease and has long been considered almost invariably present in patients experiencing AML relapse $[14,90,91]$. Notably, it has been detected at relapse even many years after the initial diagnosis, in patients experiencing more than one relapse and in relapses occurring in extramedullary sites $[14,92,93]$. However, after the first observation by Papadaki et al. that two among $21(9.5 \%)$ 
NPM1-mutated AML patients experiencing relapse, lost NPM1 mutation at leukemia recurrence [32], many groups investigating NPM1-mutated molecular MRD provided further information on stability of NPM1 mutation at relapse, as summarized in Table 2. While in several cohorts, clonal AML evolution at disease recurrence was not observed [31,33-39,42,43,48,49,52,53,55,57-60,63-69], in the remaining studies the frequencies of patients experiencing AML relapse with undetectable NPM1 mutations were extremely variable, ranging from $1 \%$ in the prospective trial by Ivey et al. [54] to $25 \%$ documented in smaller series [41,94]. Notably, cases with loss of NPM1 mutation at disease reoccurrence were initially considered as secondary therapy-related myeloid neoplasms rather than true relapses from the previously found NPM1-mutated leukemic clone [14,32,40]. Interestingly, to address the role of clonal evolution in relapsed NPM1-mutated AML, Kronke et al. applied high-resolution SNP-array profiling and performed comprehensive gene mutation screening in 53 paired BM/PB samples obtained at diagnosis and relapse [95]. High stability between primary and relapse samples was observed for mutations in DNMT3A (97\%), IDH2 (92\%) and NPM1 (91\%) genes, whereas FLT3-ITD (75\%) and IDH1 (75\%) were less stable. Interestingly, DNMT3A mutations were consistently documented in primary and relapse samples from all the 5 cases with NPM1 mutation loss at relapse, suggesting that DNMT3A mutation most likely preceded NPM1 mutation in the pathogenesis of the disease and a common ancestral DNMT3A-mutated clone with NPM1 wild-type gave rise to both primary and relapsed AML [95]. It should be noted that MRD monitoring based on RQ-PCR for NPM1 mutations may be hampered in the rare cases where relapse develops from an ancestral NPM1-negative clone, probably arising from pre-existing non-malignant clonal hematopoiesis, therefore resulting in false-negative MRD results [81,94,95]. Furthermore, a switch of the NPM1 mutation subtype from $\mathrm{D}$ to $\mathrm{A}$ has been recently described in a patient with late NPM1-mutated AML relapse, 8 years after the first diagnosis [96]. Clinical outcome of patients developing secondary AML with a switch of the NPM1 mutation subtype may be misinterpreted by follow-up strategies only focusing on rising MRD levels alone, which assume recurrence of the NPM1 mutation subtype previously documented at initial diagnosis [96]. These rare circumstances demonstrated potential limitations of RQ-PCR in MRD monitoring.

\section{Newer Molecular Technologies to Assess MRD in NPM1-Mutated AML}

A few studies have so far investigated the potential clinical application of newer molecular methods, namely ddPCR $[27,48,59,61]$ and NGS $[41,46,56,60,62,63,65,68,69,73]$, to monitor MRD in NPM1-mutated AML patients. As discussed above, at least 55 frameshift insertion NPM1 mutations have so far been recognized, and several other different gene lesions are theoretically possible, rendering relative quantification of rare NPM1 mutations by conventional RQ-PCR highly challenging [59]. Limitations of the application of RQ-PCR-based MRD assays are their dependence on specific gene mutations, requiring individual reference standard curves based on target serial dilutions, with commercial plasmid standards being widely available for only the three most common NPM1 mutation types [59,77]. ddPCR, a high-throughput technology, instead generates absolute quantification, can clonally amplify target nucleic acids, and does not require a reference standard curve $[10,77]$. More specifically, in ddPCR assays the sample is separated in a large number of partitions, and PCR reactions are compartmentalized to single oil droplets, which are then analyzed for presence or absence of a target sequence based on fluorescence. Through the use of Poisson statistics, the starting concentration of the target sequence is measured based on the number of individual reactions in which it is found to be present $[2,10,97]$. According to the first few clinical experiences in small NPM1-mutated AML patient series, ddPCR demonstrated excellent sensitivity and agreement with RQ-PCR, also allowing for the detection of a variety of rare NPM1 mutation subtypes (Tables 1 and 2) [48,59]. Notably, Mencia-Trinchant et al. created oligonucleotides with degenerate sequence at known insertion sites and combined these massively multiplex pools of insertion-specific primers into a single assay, which may selectively detect virtually all known and potential NPM1 mutations, but not wild-type NPM1 sequences [59]. Even if the precision of ddPCR assay, combined 
with its broad applicability also in patients ineligible for RQ-PCR MRD assessment, should be recognized, it should also be noted that RQ-PCR actually still performs more than adequately for MRD detection in most NPM1-mutated AML patients, is less expensive, and many clinical laboratories already possess the equipment and technical expertise for this molecular analysis [98]. In addition, primer mixes to detect multiple NPM1 mutation could also be used in RQ-PCR assays [35,37,54,99], while Mencia-Trinchant et al. do not report whether their primer mix can potentially be utilized in RQ-PCR assays [59]. However, the multiplexing of ddPCR could allow a future wider use of this molecular tool in NPM1-mutated MRD monitoring, after further validation in prospective clinical trials, at least in cases showing rarer mutations [97].

Furthermore, multi-gene NGS-based MRD monitoring has recently been applied to AML patients, including those showing NPM1 mutations (Tables 1 and 2) $[41,46,56,60,62,63,65,68,69,73]$. NGS technologies, which allow parallel and repeated sequencing of millions of small DNA fragments across many loci in order to evaluate either a few genes or an entire genome, may overcome some of the limitations of single genes analysis based on RQ-PCR assays $[10,75,77,100]$. The ability of NGS platforms to study large numbers of mutated genes could help to trace the evolution of malignant clones [77]. However, the genetic clonal heterogeneity at AML diagnosis and during the course of the disease could, conversely, complicate the MRD monitoring, because the predominant leukemic clone at presentation may not be the same clone that determines clinical relapse and mortality [77]. Moreover, intratumoral heterogeneity relies not only on the presence of different somatic mutations in distinct leukemic subclones, but also on heterogeneity of transcriptional and epigenetic states [101-103]. Unlike population-level approaches, single-cell RNA-sequencing platforms enable transcriptomic analysis of an individual cell. Through the combination of high-throughput sequencing and bioinformatic tools, single-cell RNA sequencing can detect more than 10,000 transcripts in one cell to distinguish cell subsets and dynamic cellular changes, therefore revealing intratumoral heterogeneity, with possible applications in identifying treatment-resistant leukemic cells and MRD monitoring [101-103]. Another significant limitation of NGS technology initially was the intrinsic error rate that limited its sensitivity for most single-nucleotide variants to $1-2 \%$ of all reads $[1,104]$. This notwithstanding, recent refinements of NGS technology, such as the introduction of random barcodes that anneal to target sequence fragments prior to amplification or unique molecular indexes, have eliminated the error rate of sequencing itself and markedly improved the assay sensitivity, which is actually comparable or even superior to that of RQ-PCR or MFC [75,105]. Thol et al. first conducted parallel assessment of MRD by NGS and RQ-PCR in a small series of NPM1-mutated AML patients, observing concordance in $95 \%$ of the 38 analyzed samples [41]. As mentioned above, Zhou et al. recently compared MRD testing by MFC and NGS before and after alloHSCT and showed that NGS was approximately 10-fold more sensitive than MFC, also demonstrating the clinical utility of high-sensitivity deep sequencing in risk assessment of relapse, especially after HSCT [63]. Moreover, Onecha et al. optimized and validated a high-sensitivity NGS method to detect and quantify NPM1, IDH1/2 and FLT3 mutated sequences, with survival analyses showing that MRD-positive status tested by NGS was associated with higher risk of relapse and death, whereas MRD-negativity at post-consolidation correlated with a longer DFS and OS [69]. Although NGS assay currently shows a similar sensitivity to that of RQ-PCR, it does not require oligonucleotides that hybridize specifically to a particular sequence, so all the nucleotides in the amplified region can be studied [69]. Consequently, the NGS test is capable of simultaneously detecting all NPM1 mutation types in a single assay $[41,46,56,65]$. Therefore, the application of either NGS or multiplex ddPCR may be indicated for follow-up quantification of rare NPM1 mutations subtypes, or alternatively in anecdotal cases of a switch of the NPM1 mutation subtype during the course of the disease $[69,96]$. Despite these potential advantages, NGS technology is still computationally demanding, time-consuming and expensive, so that the ELN MRD Working Party suggests that NGS techniques for MRD measurement are actually best reserved for clinical trials for further validation [74,77]. 


\section{Persistence of Pre-Leukemic Clones at AML Remission: What about NPM1 Mutations?}

In the seminal analysis of comprehensive genomic data on samples collected from 50 patients at AML presentation and remission after induction chemotherapy, Klco et al. documented persistent leukemia-associated mutations in at least $5 \%$ of BM cells at remission in $48 \%$ of cases, which were associated with increased relapse risk and reduced OS. Interestingly, contrary to other genes, NPM1 mutations were cleared below the VAF $2.5 \%$ threshold at day +30 after chemotherapy in all the 18 NPM1-mutated AML analyzed cases [106]. Accordingly, Hirsch et al. reported, in a 69-AML-patient cohort, that the detection of two or more events in $>0.4 \%$ of cells by NGS assay after one treatment course was strongly associated with lower survival in all cytogenetic profiles, especially in cases with intermediate-risk cytogenetics [107]. Furthermore, Rothenberg-Thurley et al. characterized paired pre-treatment and remission samples from 126 AML patients for mutations in 68 leukemia-associated genes. Fifty cases $(40 \%)$ retained, based on NGS analysis, at least 1 mutation during remission at a VAF of $\geq 2 \%$, with mutation persistence more frequently observed for DNMT3A (65\%), SRSF2 (64\%), TET2 (55\%) and ASXL1 (46\%), whereas NPM1 mutation was rarely persisting (1/57 patients, $2 \%$ ) [108]. Interestingly, among 46 patients with NPM1 mutation, there was a non-significant trend towards higher molecular MRD levels by RQ-PCR in patients with compared to those without persisting pre-leukemic lesions. A correlation was observed between persistence of pre-leukemic clones during first remission and inferior OS and higher incidence of relapse, which was abrogated, in this series, by alloHSCT, suggesting that mutation persistence may guide post-remission treatments [108]. More recently, further information on the prognostic role of different persisting molecular lesions in AML patients at remission has been provided [62,109]. Specifically, among 131 previously untreated AML patients, Morita et al. observed frequent persistence of somatic mutation at CR in genes that are often pre-leukemic and associated with clonal hematopoiesis of undetermined significance (CHIP), namely DNMT3A, TET2, SRSF2, ASXL1 and TP53, whereas mutations in NPM1 gene, transcription factors or receptor tyrosine kinase genes were often cleared [109]. Although the optimal VAF cut-off remains to be determined, patients who achieved mutation clearance with a VAF $<1 \%$ at day +30 after therapy, had significantly better survival and lower risk of relapse in this series. These prognostic associations were more pronounced when CHIP-related gene lesions were removed from the analysis [109]. In a larger cohort of 482 AML patients, targeted NGS found persisting gene mutations in $51.4 \%$ of patients during CR, with VAF ranging from $0.02 \%$ to $47 \%$. The detection of persistent mutations in DNMT3A, TET2 and ASXL1 genes (DTA mutations) was not correlated with an increased relapse rate. Only after exclusion of DTA mutations from the analysis, the documentation of molecular MRD display significantly higher relapse rates and lower survival rates, thereby confirming that persistent mutations in genes associated with age-related CHIP did not have prognostic value in adult patients within a 4-year time frame [62]. Overall, these studies provided information on the use of a genome-wide approach to evaluate the impact of persistent multiple gene mutations on treatment outcomes [62,106-109]. The monitoring of specific single-gene MRD levels, including quantification of NPM1 transcripts generally absent in pre-leukemic clones, could potentially be integrated with evaluation of persisting pre-leukemic molecular lesions at remission. The combination of these two variables may ultimately provide complimentary prognostic information and it could potentially be argued that monitoring both leukemic and pre-leukemic clones after therapy could, in perspective, complement or even supersede pre-treatment genetic factors for AML prognostic stratification [108].

\section{Conclusions}

Even though several published clinical studies (Tables 1 and 2) support the independent prognostic significance of molecular MRD monitoring in NPM1-mutated AML patients, and the Consensus Document from ELN MRD Working Party clearly indicates that patients showing NPM1 mutations should have molecular assessment of MRD, we have discussed that several controversies have been raised, and further prospective clinical trials are warranted to standardize the optimal material, timepoints, molecular tools and cut-offs for a meaningful application of MRD monitoring 
to inform treatment decisions $[31,74,110]$. Caution is still needed when using MRD results alone, outside of a clinical trial, to make decisions with regard to treatment approaches with potentially high morbidity and mortality, mainly alloHSCT, in otherwise genetically favorable-risk AML patients [8]. Interestingly, the application of pre-emptive, MRD-directed treatment strategies, potentially including pharmacological agents targeting NPM1-mutated protein with induction of protein degradation or drugs inhibiting NPM1 cytoplasmic translocation, such as selinexor, which acts through inhibition of nuclear export and resulting in NPM1 protein nuclear relocalization, should be warranted and needs to be prospectively investigated [111-113].

Author Contributions: F.F. designed the study, reviewed the literature and wrote the manuscript; P.C., R.M., L.P. and M.L. supervised the study, analyzed data and critically revised the manuscript. All authors approved the final version of the manuscript.

Funding: This work was supported by grants from the Associazione Italiana per la Ricerca sul Cancro (AIRC), Milan, Italy (IG 14797-2013) (ML); Fondazione Istituto di Ricovero e Cura a Carattere Scientifico (IRCCS) Policlinico San Matteo [Ricerca Corrente 08069113 (PC)].

Acknowledgments: We would like to thank Chiara Quadrelli (RIGENERAND srl. Medolla, Italy) for her technical support during development of RQ-PCR assay for NPM1-mutated transcripts and Rossana Maffei, Silvia Martinelli and Patrizia Zucchini, for implementing and validating this assay and other diagnostic molecular techniques for hematologic malignancies, at our Institution.

Conflicts of Interest: F.F. served on advisory boards for Jannsen on the clinical use of decitabine in AML patients, M.L. served on advisory boards for Novartis on the clinical use of midostaurin. The other authors declare no potential conflicts of interest.

\section{References}

1. Tomlinson, B.; Lazarus, H.M. Enhancing acute myeloid leukemia therapy-Monitoring response using residual disease testing as a guide to therapeutic decision-making. Expert Rev. Hematol. 2017, 10, 563-574. [CrossRef] [PubMed]

2. Ossenkoppele, G.; Schuurhuis, G.J. MRD in AML: Does it already guide therapy decision-making? Hematol. Am. Soc. Hematol. Educ. Program 2016, 2016, 356-365. [CrossRef] [PubMed]

3. Hourigan, C.S.; Gale, R.P.; Gormley, N.J.; Ossenkoppele, G.J.; Walter, R.B. Measurable residual disease testing in acute myeloid leukemia. Leukemia 2017, 31, 1482-1490. [CrossRef] [PubMed]

4. Buccisano, F.; Maurillo, L.; Del Principe, M.I.; Di Veroli, A.; De Bellis, E.; Biagi, A.; Zizzari, A.; Rossi, V.; Rapisarda, V.; Amadori, S.; et al. Minimal residual disease as a biomarker for outcome prediction and therapy optimization in acute myeloid leukemia. Expert Rev. Hematol. 2018, 11, 307-313. [CrossRef] [PubMed]

5. Araki, D.; Wood, B.L.; Othus, M.; Radich, J.P.; Halpern, A.B.; Zhou, Y.; Mielcarek, M.; Estey, E.H.; Appelbaum, F.R.; Walter, R.B. Allogeneic hematopoietic cell transplantation for acute myeloid leukemia: Time to move toward a minimal residual disease-based definition of complete remission? J. Clin. Oncol. 2016, 34, 329-336. [CrossRef] [PubMed]

6. Inaba, H.; Coustan-Smith, E.; Cao, X.; Pounds, S.B.; Shurtleff, S.A.; Wang, K.Y.; Raimondi, S.C.; Onciu, M.; Jacobsen, J.; Ribeiro, R.C.; et al. Comparative analysis of different approaches to measure response in acute myeloid leukemia. J. Clin. Oncol. 2012, 30, 3625-3632. [CrossRef] [PubMed]

7. Dohner, H.; Estey, E.; Grimwade, D.; Amadori, S.; Appelbaum, F.R.; Buchner, T.; Dombret, H.; Ebert, B.L.; Fenaux, P.; Larson, R.A.; et al. Diagnosis and management of AML in adults: 2017 ELN recommendations from an international expert panel. Blood 2017, 129, 424-447. [CrossRef] [PubMed]

8. Mosna, F.; Capelli, D.; Gottardi, M. Minimal residual disease in acute myeloid leukemia: Still a work in progress? J. Clin. Med. 2017, 6, 57. [CrossRef] [PubMed]

9. Grimwade, D.; Freeman, S.D. Defining minimal residual disease in acute myeloid leukemia: Which platforms are ready for "prime time"? Blood 2014, 124, 3345-3355. [CrossRef] [PubMed]

10. Coltoff, A.; Houldsworth, J.; Keyzner, A.; Renteria, A.S.; Mascarenhas, J. Role of minimal residual disease in the management of acute myeloid leukemia-A case-based discussion. Ann. Hematol. 2018, 97, 1155-1167. [CrossRef] [PubMed]

11. Grisendi, S.; Mecucci, C.; Falini, B.; Pandolfi, P.P. Nucleophosmin and cancer. Nat. Rev. Cancer 2006, 6, 493-505. [CrossRef] [PubMed] 
12. Falini, B.; Nicoletti, I.; Martelli, M.F.; Mecucci, C. Acute myeloid leukemia carrying cytoplasmic/mutated nucleophosmin (NPMc+ AML): Biologic and clinical features. Blood 2007, 109, 874-885. [CrossRef] [PubMed]

13. Falini, B.; Bolli, N.; Liso, A.; Martelli, M.P.; Mannucci, R.; Pileri, S.; Nicoletti, I. Altered nucleophosmin transport in acute myeloid leukaemia with mutated NPM1: Molecular basis and clinical implications. Leukemia 2009, 23, 1731-1743. [CrossRef] [PubMed]

14. Falini, B.; Martelli, M.P.; Bolli, N.; Sportoletti, P.; Liso, A.; Tiacci, E.; Haferlach, T. Acute myeloid leukemia with mutated nucleophosmin (NPM1): Is it a distinct entity? Blood 2011, 117, 1109-1120. [CrossRef] [PubMed]

15. Grimwade, D.; Ivey, A.; Huntly, B.J.P. Molecular landscape of acute myeloid leukemia in younger adults and its clinical relevance. Blood 2016, 127, 29-41. [CrossRef] [PubMed]

16. Falini, B.; Mecucci, C.; Tiacci, E.; Alcalay, M.; Rosati, R.; Pasqualucci, L.; La Starza, R.; Diverio, D.; Colombo, E.; Santucci, A.; et al. Cytoplasmic nucleophosmin in acute myelogenous leukemia with a normal karyotype. N. Engl. J. Med. 2005, 352, 254-266. [CrossRef] [PubMed]

17. Heath, E.M.; Chan, S.M.; Minden, M.D.; Murphy, T.; Shlush, L.I.; Schimmer, A.D. Biological and clinical consequences of NPM1 mutations in AML. Leukemia 2017, 31, 798-807. [CrossRef] [PubMed]

18. Falini, B.; Martelli, M.P.; Bolli, N.; Bonasso, R.; Ghia, E.; Pallotta, M.T.; Diverio, D.; Nicoletti, I.; Pacini, R.; Tabarrini, A.; et al. Immunohistochemistry predicts nucleophosmin (NPM) mutations in acute myeloid leukemia. Blood 2006, 108, 1999-2005. [CrossRef] [PubMed]

19. Gu, X.; Ebrahem, Q.; Mahfouz, R.Z.; Hasipek, M.; Enane, F.; Radivoyevitch, T.; Rapin, N.; Przychodzen, B.; $\mathrm{Hu}, \mathrm{Z}$; Balusu, R.; et al. Leukemogenic nucleophosmin mutation disrupts the transcription factor hub regulating granulo-monocytic fates. J. Clin. Investig. 2018, 128, 4260-4279. [CrossRef] [PubMed]

20. Forghieri, F.; Paolini, A.; Morselli, M.; Bigliardi, S.; Bonacorsi, G.; Leonardi, G.; Coluccio, V.; Maccaferri, M.; Fantuzzi, V.; Faglioni, L.; et al. NPM1 mutations may reveal acute myeloid leukemia in cases otherwise morphologically diagnosed as myelodysplastic syndromes or myelodysplastic/myeloproliferative neoplasms. Leuk. Lymphoma 2015, 56, 3222-3226. [CrossRef] [PubMed]

21. Arber, D.A.; Orazi, A.; Hasserjian, R.; Thiele, J.; Borowitz, M.J.; Le Beau, M.M.; Bloomfield, C.D.; Cazzola, M.; Vardiman, J.W. The 2016 revision to the World Health Organization classification of myeloid neoplasms and acute leukemia. Blood 2016, 127, 2391-2405. [CrossRef] [PubMed]

22. Haferlach, C.; Mecucci, C.; Schnittger, S.; Kohlmann, A.; Mancini, M.; Cuneo, A.; Testoni, N.; Rege-Cambrin, G.; Santucci, A.; Vignetti, M.; et al. AML with mutated NPM1 carrying a normal or aberrant karyotype show overlapping biologic, pathologic, immunophenotypic, and prognostic features. Blood 2009, 114, 3024-3032. [CrossRef] [PubMed]

23. Micol, J.B.; Boissel, N.; Renneville, A.; Castaigne, S.; Gardin, C.; Preudhomme, C.; Dombret, H. The role of cytogenetic abnormalities in acute myeloid leukemia with NPM1 mutations and no FLT3 internal tandem duplication. Blood 2009, 114, 4601-4602. [CrossRef] [PubMed]

24. Döhner, K.; Schlenk, R.F.; Habdank, M.; Scholl, C.; Rücker, F.G.; Corbacioglu, A.; Bullinger, L.; Fröhling, S.; Döhner, H. Mutant nucleophosmin (NPM1) predicts favorable prognosis in younger adults with acute myeloid leukemia and normal cytogenetics: Interaction with other gene mutations. Blood 2005, 106, 3740-3746. [CrossRef] [PubMed]

25. Papaemmanuil, E.; Gerstung, M.; Bullinger, L.; Gaidzik, V.I.; Paschka, P.; Roberts, N.D.; Potter, N.E.; Heuser, M.; Thol, F.; Bolli, N.; et al. Genomic classification and prognosis in acute myeloid leukemia. N. Engl. J. Med. 2016, 374, 2209-2221. [CrossRef] [PubMed]

26. Gale, R.E.; Green, C.; Allen, C.; Mead, A.J.; Burnett, A.K.; Hills, R.K.; Linch, D.C.; Medical Research Council Adult Leukaemia Working Party. The impact of FLT3 internal tandem duplication mutant level, number, size, and interaction with NPM1 mutations in a large cohort of young adult patients with acute myeloid leukemia. Blood 2008, 111, 2776-2784. [CrossRef] [PubMed]

27. Pratcorona, M.; Brunet, S.; Nomdedéu, J.; Ribera, J.M.; Tormo, M.; Duarte, R.; Escoda, L.; Guàrdia, R.; Queipo de Llano, M.P.; Salamero, O.; et al. Favorable outcome of patients with acute myeloid leukemia harboring a low-allelic burden FLT3-ITD mutation and concomitant NPM1 mutation: Relevance to postremission therapy. Blood 2013, 121, 2734-2738. [CrossRef] [PubMed]

28. Schlenk, R.F.; Kayser, S.; Bullinger, L.; Kobbe, G.; Casper, J.; Ringhoffer, M.; Held, G.; Brossart, P.; Lübbert, M.; Salih, H.R.; et al. Differential impact of allelic ratio and insertion site in FLT3-ITD positive AML with respect to allogeneic transplantation. Blood 2014, 124, 3441-3449. [CrossRef] [PubMed] 
29. Linch, D.C.; Hills, R.K.; Burnett, A.K.; Khwaja, A.; Gale, R.E. Impact of FLT3(ITD) mutant allele level on relapse risk in intermediate-risk acute myeloid leukemia. Blood 2014, 124, 273-276. [CrossRef] [PubMed]

30. Gorello, P.; Cazzaniga, G.; Alberti, F.; Dell'Oro, M.G.; Gottardi, E.; Specchia, G.; Roti, G.; Rosati, R.; Martelli, M.F.; Diverio, D.; et al. Quantitative assessment of minimal residual disease in acute myeloid leukemia carrying nucleophosmin (NPM1) gene mutations. Leukemia 2007, 21, 340-350. [CrossRef] [PubMed]

31. Chou, W.C.; Tang, J.L.; Wu, S.J.; Tsay, W.; Yao, M.; Huang, S.Y.; Huang, K.C.; Chen, C.Y.; Huang, C.F.; Tien, H.F. Clinical implications of minimal residual disease monitoring by quantitative polymerase chain reaction in acute myeloid leukemia patients bearing nucleophosmin (NPM1) mutations. Leukemia 2007, 21, 998-1004. [CrossRef] [PubMed]

32. Papadaki, C.; Dufour, A.; Seibl, M.; Schneider, S.; Bohlander, S.K.; Zellmeier, E.; Mellert, G.; Hiddemann, W.; Spiekermann, K. Monitoring minimal residual disease in acute myeloid leukaemia with NPM1 mutations by quantitative PCR: Clonal evolution is a limiting factor. Br. J. Haematol. 2009, 144, 517-523. [CrossRef] [PubMed]

33. Barragan, E.; Pajuelo, J.C.; Ballester, S.; Fuster, O.; Cervera, J.; Moscardo, F.; Senent, L.; Such, E.; Sanz, M.A.; Bolufer, P. Minimal residual disease detection in acute myeloid leukemia by mutant nucleophosmin (NPM1): Comparison with WT1 gene expression. Clin. Chim. Acta 2008, 395, 120-123. [CrossRef] [PubMed]

34. Bacher, U.; Badbaran, A.; Fehse, B.; Zabelina, T.; Zander, A.R.; Kröger, N. Quantitative monitoring of NPM1 mutations provides a valid minimal residual disease parameter following allogeneic stem cell transplantation. Exp. Hematol. 2009, 37, 135-142. [CrossRef] [PubMed]

35. Schnittger, S.; Kern, W.; Tschulik, C.; Weiss, T.; Dicker, F.; Falini, B.; Haferlach, C.; Haferlach, T. Minimal residual disease levels assessed by NPM1 mutation-specific RQ-PCR provide important prognostic information in AML. Blood 2009, 114, 2220-2231. [CrossRef] [PubMed]

36. Stahl, T.; Badbaran, A.; Kröger, N.; Klyuchnikov, E.; Zabelina, T.; Zeschke, S.; Schafhausen, P.; Schultz, W.; Asenova, S.; Smirnova, A.; et al. Minimal residual disease diagnostics in patients with acute myeloid leukemia in the post-transplant period: Comparison of peripheral blood and bone marrow analysis. Leuk. Lymphoma 2010, 51, 1837-1843. [CrossRef] [PubMed]

37. Dvorakova, D.; Racil, Z.; Jeziskova, I.; Palasek, I.; Protivankova, M.; Lengerova, M.; Razga, F.; Mayer, J. Monitoring of minimal residual disease in acute myeloid leukemia with frequent and rare patient-specific NPM1 mutations. Am. J. Hematol. 2010, 85, 926-929. [CrossRef] [PubMed]

38. Ommen, H.B.; Schnittger, S.; Jovanovic, J.V.; Ommen, I.B.; Hasle, H.; Østergaard, M.; Grimwade, D.; Hokland, P. Strikingly different molecular relapse kinetics in NPM1c, PML-RARA, RUNX1-RUNX1T1, and CBFB-MYH11 acute myeloid leukemias. Blood 2010, 115, 198-205. [CrossRef] [PubMed]

39. Kristensen, T.; Møller, M.B.; Friis, L.; Bergmann, O.J.; Preiss, B. NPM1 mutation is a stable marker for minimal residual disease monitoring in acute myeloid leukaemia patients with increased sensitivity compared to WT1 expression. Eur. J. Haematol. 2011, 87, 400-408. [CrossRef] [PubMed]

40. Krönke, J.; Schlenk, R.F.; Jensen, K.O.; Tschürtz, F.; Corbacioglu, A.; Gaidzik, V.I.; Paschka, P.; Onken, S.; Eiwen, K.; Habdank, M.; et al. Monitoring of minimal residual disease in NPM1-mutated acute myeloid leukemia: A study from the German-Austrian acute myeloid leukemia study group. J. Clin. Oncol. 2011, 29, 2709-2716. [CrossRef] [PubMed]

41. Thol, F.; Kölking, B.; Damm, F.; Reinhardt, K.; Klusmann, J.H.; Reinhardt, D.; von Neuhoff, N.; Brugman, M.H.; Schlegelberger, B.; Suerbaum, S.; et al. Next-generation sequencing for minimal residual disease monitoring in acute myeloid leukemia patients with FLT3-ITD or NPM1 mutations. Genes Chromosomes Cancer 2012, 51, 689-695. [CrossRef] [PubMed]

42. Abdelhamid, E.; Preudhomme, C.; Helevaut, N.; Nibourel, O.; Gardin, C.; Rousselot, P.; Castaigne, S.; Gruson, B.; Berthon, C.; Soua, Z.; et al. Minimal residual disease monitoring based on FLT3 internal tandem duplication in adult acute myeloid leukemia. Leuk. Res. 2012, 36, 316-323. [CrossRef] [PubMed]

43. Schiller, J.; Praulich, I.; Krings Rocha, C.; Kreuzer, K.A. Patient-specific analysis of FLT3 internal tandem duplications for the prognostication and monitoring of acute myeloid leukemia. Eur. J. Haematol. 2012, 89, 53-62. [CrossRef] [PubMed]

44. Shayegi, N.; Kramer, M.; Bornhäuser, M.; Schaich, M.; Schetelig, J.; Platzbecker, U.; Röllig, C.; Heiderich, C.; Landt, O.; Ehninger, G.; et al. The level of residual disease based on mutant NPM1 is an independent prognostic factor for relapse and survival in AML. Blood 2013, 122, 83-92. [CrossRef] [PubMed] 
45. Jeziskova, I.; Razga, F.; Toskova, M.; Dvorakova, D.; Timilsina, S.; Mayer, J.; Racil, Z. Quantitative detection of IDH2 mutation for minimal residual disease monitoring in patients with acute myeloid leukemia and its comparison with mutations in NPM1 gene. Leuk. Lymphoma 2013, 54, 867-870. [CrossRef] [PubMed]

46. Salipante, S.J.; Fromm, J.R.; Shendure, J.; Wood, B.L.; Wu, D. Detection of minimal residual disease in NPM1-mutated acute myeloid leukemia by next-generation sequencing. Mod. Pathol. 2014, 27, 1438-1446. [CrossRef] [PubMed]

47. Hubmann, M.; Köhnke, T.; Hoster, E.; Schneider, S.; Dufour, A.; Zellmeier, E.; Fiegl, M.; Braess, J.; Bohlander, S.K.; Subklewe, M.; et al. Molecular response assessment by quantitative real-time polymerase chain reaction after induction therapy in NPM1-mutated patients identifies those at high risk of relapse. Haematologica 2014, 99, 1317-1325. [CrossRef] [PubMed]

48. Bacher, U.; Dicker, F.; Haferlach, C.; Alpermann, T.; Rose, D.; Kern, W.; Haferlach, T.; Schnittger, S. Quantification of rare NPM1 mutation subtypes by digital PCR. Br. J. Haematol. 2014, 167, 710-714. [CrossRef] [PubMed]

49. Lambert, J.; Lambert, J.; Nibourel, O.; Pautas, C.; Hayette, S.; Cayuela, J.M.; Terré, C.; Rousselot, P.; Dombret, H.; Chevret, S.; et al. MRD assessed by WT1 and NPM1 transcript levels identifies distinct outcomes in AML patients and is influenced by gemtuzumab ozogamicin. Oncotarget 2014, 5, 6280-6288. [CrossRef] [PubMed]

50. Debarri, H.; Lebon, D.; Roumier, C.; Cheok, M.; Marceau-Renaut, A.; Nibourel, O.; Geffroy, S.; Helevaut, N.; Rousselot, P.; Gruson, B.; et al. IDH1/2 but not DNMT3A mutations are suitable targets for minimal residual disease monitoring in acute myeloid leukemia patients: A study by the Acute Leukemia French Association. Oncotarget 2015, 6, 42345-42353. [CrossRef] [PubMed]

51. Pettersson, L.; Levéen, P.; Axler, O.; Dvorakova, D.; Juliusson, G.; Ehinger, M. Improved minimal residual disease detection by targeted quantitative polymerase chain reaction in Nucleophosmin 1 type a mutated acute myeloid leukemia. Genes Chromosomes Cancer 2016, 55, 750-766. [CrossRef] [PubMed]

52. Karas, M.; Steinerova, K.; Lysak, D.; Hrabetova, M.; Jungova, A.; Sramek, J.; Jindra, P.; Polivka, J.; Holubec, L. Pre-transplant quantitative determination of NPM1 mutation significantly predicts outcome of allogeneic hematopoietic stem cell transplantation in patients with normal karyotype AML in complete remission. Anticancer Res. 2016, 36, 5487-5498. [CrossRef] [PubMed]

53. Alizad Ghandforoush, N.; Chahardouli, B.; Rostami, S.; Ghadimi, H.; Ghasemi, A.; Alimoghaddam, K.; Ghavamzadeh, A.; Nadali, F. Evaluation of minimal residual disease in acute myeloid leukemia with NPM1 marker. Int. J. Hematol. Oncol. Stem Cell. Res. 2016, 10, 147-152. [PubMed]

54. Ivey, A.; Hills, R.K.; Simpson, M.A.; Jovanovic, J.V.; Gilkes, A.; Grech, A.; Patel, Y.; Bhudia, N.; Farah, H.; Mason, J.; et al. Assessment of minimal residual disease in standard-risk AML. N. Engl. J. Med. 2016, 374, 422-433. [CrossRef] [PubMed]

55. Kayser, S.; Benner, A.; Thiede, C.; Martens, U.; Huber, J.; Stadtherr, P.; Janssen, J.W.; Röllig, C.; Uppenkamp, M.J.; Bochtler, T.; et al. Pretransplant NPM1 MRD levels predict outcome after allogeneic hematopoietic stem cell transplantation in patients with acute myeloid leukemia. Blood Cancer J. 2016, 6, e449. [CrossRef] [PubMed]

56. Malmberg, E.B.; Ståhlman, S.; Rehammar, A.; Samuelsson, T.; Alm, S.J.; Kristiansson, E.; Abrahamsson, J.; Garelius, H.; Pettersson, L.; Ehinger, M.; et al. Patient-tailored analysis of minimal residual disease in acute myeloid leukemia using next-generation sequencing. Eur. J. Haematol. 2017, 98, 26-37. [CrossRef] [PubMed]

57. Balsat, M.; Renneville, A.; Thomas, X.; de Botton, S.; Caillot, D.; Marceau, A.; Lemasle, E.; Marolleau, J.P.; Nibourel, O.; Berthon, C.; et al. Postinduction minimal residual disease predicts outcome and benefit from allogeneic stem cell transplantation in acute myeloid leukemia with NPM1 mutation: A study by the Acute Leukemia French Association Group. J. Clin. Oncol. 2017, 35, 185-193. [CrossRef] [PubMed]

58. Schieppati, F.; Passi, A.; Borlenghi, E.; Lamorgese, C.; Ruggeri, G.; Chiarini, M.; Giustini, V.; Malagola, M.; Cattaneo, C.; Sciumé, M.; et al. Molecular monitoring of NPM1 is a useful tool for detecting early-stage relapse and to guide pre-emptive treatment approaches in patients with NPM1-mutated acute myeloid leukemia. Blood 2017, 130, 3931.

59. Mencia-Trinchant, N.; Hu, Y.; Alas, M.A.; Ali, F.; Wouters, B.J.; Lee, S.; Ritchie, E.K.; Desai, P.; Guzman, M.L.; Roboz, G.J.; et al. Minimal residual disease monitoring of acute myeloid leukemia by massively multiplex digital PCR in patients with NPM1 mutations. J. Mol. Diagn. 2017, 19, 537-548. [CrossRef] [PubMed] 
60. Getta, B.M.; Devlin, S.M.; Levine, R.L.; Arcila, M.E.; Mohanty, A.S.; Zehir, A.; Tallman, M.S.; Giralt, S.A.; Roshal, M. Multicolor flow cytometry and multigene Next-Generation Sequencing are complementary and highly predictive for relapse in acute myeloid leukemia after allogeneic transplantation. Biol. Blood Marrow Transplant. 2017, 23, 1064-1071. [CrossRef] [PubMed]

61. Bill, M.; Grimm, J.; Jentzsch, M.; Kloss, L.; Goldmann, K.; Schulz, J.; Beinicke, S.; Häntschel, J.; Cross, M.; Vucinic, V.; et al. Digital droplet PCR-based absolute quantification of pre-transplant NPM1 mutation burden predicts relapse in acute myeloid leukemia patients. Ann. Hematol. 2018, 97, 1757-1765. [CrossRef] [PubMed]

62. Jongen-Lavrencic, M.; Grob, T.; Hanekamp, D.; Kavelaars, F.G.; Al Hinai, A.; Zeilemaker, A.; Erpelinck-Verschueren, C.A.J.; Gradowska, P.L.; Meijer, R.; Cloos, J.; et al. Molecular minimal residual disease in acute myeloid leukemia. N. Engl. J. Med. 2018, 378, 1189-1199. [CrossRef] [PubMed]

63. Zhou, Y.; Othus, M.; Walter, R.B.; Estey, E.H.; Wu, D.; Wood, B.L. Deep NPM1 sequencing following allogeneic hematopoietic cell transplantation improves risk assessment in adults with NPM1-mutated AML. Biol. Blood Marrow Transplant. 2018, 24, 1615-1620. [CrossRef] [PubMed]

64. Zappasodi, P.; Marbello, L.; Borlenghi, E.; Fumagalli, M.; Bernardi, M.; Fracchiolla, N.; Mancini, V.; Da Vià, M.; Ravano, E.; Cerqui, E.; et al. Molecular remission at the end of treatment is a necessary goal for a good outcome in ELN favorable-risk acute myeloid leukemia: A real-life analysis on 201 patients by the Rete Ematologica Lombarda network. Ann. Hematol. 2018. [CrossRef] [PubMed]

65. Delsing Malmberg, E.; Johansson Alm, S.; Nicklasson, M.; Lazarevic, V.; Ståhlman, S.; Samuelsson, T.; Lenhoff, S.; Asp, J.; Ehinger, M.; Palmqvist, L.; et al. Minimal residual disease assessed with deep sequencing of NPM1 mutations predicts relapse after allogeneic stem cell transplant in AML. Leuk. Lymphoma 2018. [CrossRef] [PubMed]

66. Kapp-Schwoerer, S.; Corbacioglu, A.; Weber, D.; Gaidzik, V.I.; Rucker, F.G.; Paschka, P.; Kronke, J.; Teleanu, M.V.; Gohring, G.; Schlegelberger, B.; et al. Impact of NPM1 MRD status after two cycles of intensive chemotherapy to inform type of post-remission therapy: A study of the AML study group (AMLSG). HemaSphere 2018, 2, 434.

67. Caprioli, C.; Lussana, F.; Stefanoni, P.; Pavoni, C.; Spinelli, O.; Buklijas, K.; Michelato, A.; Zanghì, P.; Borleri, G.; Algarotti, A.; et al. Comparison between multiparametric flow cytometry and RT-QPCR assay in evaluating minimal residual disease before allogeneic stem cell transplantation in NPM1 acute myeloid leukemia. HemaSphere 2018, 2, 443.

68. Patkar, N.; Kodgule, R.; Kakirde, C.; Raval, G.; Bhanshe, P.; Badrinath, Y.; Ghoghale, S.; Kadechkar, S.; Khizer, S.H.; Kannan, S.; et al. Detection of measurable residual disease (MRD) by ultradeep next generation sequencing (NGS) is highly predictive of outcome in NPM1 mutated acute myeloid leukemia (AML). HemaSphere 2018, 2, 55.

69. Onecha, E.; Linares, M.; Rapado, I.; Ruiz-Heredia, Y.; Martinez-Sanchez, P.; Cedena, T.; Pratcorona, M.; Perez Oteyza, J.; Herrera, P.; Barragan, E.; et al. Novel deep targeted sequencing method for minimal residual disease monitoring in acute myeloid leukemia. Haematologica 2018. [CrossRef] [PubMed]

70. Petrova, L.; Vrbacky, F.; Lanska, M.; Zavrelova, A.; Zak, P.; Hrochova, K. IDH1 and IDH2 mutations in patients with acute myeloid leukemia: Suitable targets for minimal residual disease monitoring? Clin. Biochem. 2018. [CrossRef] [PubMed]

71. Prata, P.H.; Bally, C.; Prebet, T.; Recher, C.; Venton, G.; Thomas, X.; Raffoux, E.; Pigneux, A.; Cluzeau, T.; Desoutter, J.; et al. NPM1 mutation is not associated with prolonged complete remission in acute myeloid leukemia patients treated with hypomethylating agents. Haematologica 2018, 103, e455-e457. [CrossRef] [PubMed]

72. Ottone, T.; Alfonso, V.; Iaccarino, L.; Hasan, S.K.; Mancini, M.; Divona, M.; Lavorgna, S.; Cicconi, L.; Panetta, P.; Maurillo, L.; et al. Longitudinal detection of DNMT3A ${ }^{\mathrm{R} 822 \mathrm{H}}$ transcripts in patients with acute myeloid leukemia. Am. J. Hematol. 2018, 93, e120-e123. [CrossRef] [PubMed]

73. Gaksch, L.; Kashofer, K.; Heitzer, E.; Quehenberger, F.; Daga, S.; Hofer, S.; Halbwedl, I.; Graf, R.; Krisper, N.; Hoefler, G.; et al. Residual disease detection using parallel sequencing predicts relapse in cytogenetically normal acute myeloid leukemia. Am. J. Hematol. 2018, 93, 23-30. [CrossRef] [PubMed]

74. Schuurhuis, G.J.; Heuser, M.; Freeman, S.; Béné, M.C.; Buccisano, F.; Cloos, J.; Grimwade, D.; Haferlach, T.; Hills, R.K.; Hourigan, C.S.; et al. Minimal/measurable residual disease in AML: A consensus document from the European LeukemiaNet MRD Working Party. Blood 2018, 131, 1275-1291. [CrossRef] [PubMed] 
75. Hantel, A.; Stock, W.; Kosuri, S. Molecular minimal residual disease testing in acute myeloid leukemia: A review for the practicing clinician. Clin. Lymphoma Myeloma Leuk. 2018, 18, 636-647. [CrossRef] [PubMed]

76. Patel, S.S.; Kuo, F.C.; Gibson, C.J.; Steensma, D.P.; Soiffer, R.J.; Alyea, E.P.; Chen, Y.A.; Fathi, A.T.; Graubert, T.A.; Brunner, A.M.; et al. High NPM1-mutant allele burden at diagnosis predicts unfavorable outcomes in de novo AML. Blood 2018, 131, 2816-2825. [CrossRef] [PubMed]

77. Ravandi, F.; Walter, R.B.; Freeman, S.D. Evaluating measurable residual disease in acute myeloid leukemia. Blood Adv. 2018, 2, 1356-1366. [PubMed]

78. Röllig, C.; Bornhäuser, M.; Kramer, M.; Thiede, C.; Ho, A.D.; Krämer, A.; Schäfer-Eckart, K.; Wandt, H.; Hänel, M.; Einsele, H.; et al. Allogeneic stem-cell transplantation in patients with NPM1-mutated acute myeloid leukemia: Results from a prospective donor versus no-donor analysis of patients after upfront HLA typing within the SAL-AML 2003 trial. J. Clin. Oncol. 2015, 33, 403-410. [CrossRef] [PubMed]

79. Lee, C.J.; Savani, B.N.; Mohty, M.; Gorin, N.C.; Labopin, M.; Ruggeri, A.; Schmid, C.; Baron, F.; Esteve, J.; Giebel, S.; et al. Post-remission strategies for the prevention of relapse following allogeneic hematopoietic cell transplantation for high-risk acute myeloid leukemia: Expert review from the Acute Leukemia Working Party of the European Society for Blood and Marrow Transplantation. Bone Marrow Transplant. 2018. [CrossRef]

80. Platzbecker, U.; Wermke, M.; Radke, J.; Oelschlaegel, U.; Seltmann, F.; Kiani, A.; Klut, I.M.; Knoth, H.; Röllig, C.; Schetelig, J.; et al. Azacitidine for treatment of imminent relapse in MDS or AML patients after allogeneic HSCT: Results of the RELAZA trial. Leukemia 2012, 26, 381-389. [CrossRef] [PubMed]

81. Platzbecker, U.; Middeke, J.M.; Sockel, K.; Mutherig, A.; Herbst, R.; Hanel, M.; Wolf, D.; Baldus, C.D.; Fransecky, L.; Noppeney, R.; et al. Minimal-residual disease guided treatment with azacitidine in MDS/AML patients at imminent risk of relapse: Results of the prospective RELAZA2 trial. Blood 2017, 130, 565.

82. Sockel, K.; Wermke, M.; Radke, J.; Kiani, A.; Schaich, M.; Bornhäuser, M.; Ehninger, G.; Thiede, C.; Platzbecker, U. Minimal residual disease-directed preemptive treatment with azacitidine in patients with NPM1-mutant acute myeloid leukemia and molecular relapse. Haematologica 2011, 96, 1568-1570. [CrossRef] [PubMed]

83. Buccisano, F.; Dillon, R.; Freeman, S.D.; Venditti, A. Role of minimal (measurable) residual disease assessment in older patients with acute myeloid leukemia. Cancers 2018, 10, 215. [CrossRef] [PubMed]

84. Siegel, R.; Ma, J.; Zou, Z.; Jemal, A. Cancer statistics, 2014. CA Cancer J. Clin. 2014, 64, 9-29. [CrossRef] [PubMed]

85. Arellano, M.; Carlisle, J.W. How I treat older patients with acute myeloid leukemia. Cancer 2018, 124, 2472-2483. [CrossRef] [PubMed]

86. Dombret, H.; Gardin, C. An update of current treatments for adult acute myeloid leukemia. Blood 2016, 127, 53-61. [CrossRef] [PubMed]

87. Bullinger, L.; Dohner, K.; Dohner, H. Genomics of acute myeloid leukemia diagnosis and pathways. J. Clin. Oncol. 2017, 35, 934-946. [CrossRef] [PubMed]

88. Ostronoff, F.; Othus, M.; Lazenby, M.; Estey, E.; Appelbaum, F.R.; Evans, A.; Godwin, J.; Gilkes, A.; Kopecky, K.J.; Burnett, A.; et al. Prognostic significance of NPM1 mutations in the absence of FLT3-Internal tandem duplication in older patients with acute myeloid leukemia: A SWOG and UK National Cancer Research Institute/Medical Research Council report. J. Clin. Oncol. 2015, 33, 1157-1164. [CrossRef] [PubMed]

89. Prassek, V.V.; Rothenberg-Thurley, M.; Sauerland, M.C.; Herold, T.; Janke, H.; Ksienzyk, B.; Konstandin, N.P.; Goerlich, D.; Krug, U.; Faldum, A.; et al. Genetics of acute myeloid leukemia in the elderly: Mutation spectrum and clinical impact in intensively treated patients aged $\geq 75$ years. Haematologica 2018, 103, 1853-1861. [CrossRef] [PubMed]

90. Chou, W.C.; Tang, J.L.; Lin, L.I.; Yao, M.; Tsay, W.; Chen, C.Y.; Wu, S.J.; Huang, C.F.; Chiou, R.J.; Tseng, M.H.; et al. Nucleophosmin mutations in de novo acute myeloid leukemia: The age-dependent incidences and the stability during disease evolution. Cancer Res. 2006, 66, 3310-3316. [CrossRef] [PubMed]

91. Falini, B.; Martelli, M.P.; Mecucci, C.; Liso, A.; Bolli, N.; Bigerna, B.; Pucciarini, A.; Pileri, S.; Meloni, G.; Martelli, M.F.; et al. Cytoplasmic mutated nucleophosmin is stable in primary leukemic cells and in a xenotransplant model of NPMc_acute myeloid leukemia in SCID mice. Haematologica 2008, 93, 775-779. [CrossRef] [PubMed]

92. Meloni, G.; Mancini, M.; Gianfelici, V.; Martelli, M.P.; Foa, R.; Falini, B. Late relapse of acute myeloid leukemia with mutated NPM1 after eight years: Evidence of NPM1 mutation stability. Haematologica 2009, 94, $298-300$. [CrossRef] [PubMed] 
93. Bolli, N.; Galimberti, S.; Martelli, M.P.; Tabarrini, A.; Roti, G.; Mecucci, C.; Martelli, M.F.; Petrini, M.; Falini, B. Cytoplasmic nucleophosmin in myeloid sarcoma occurring 20 years after diagnosis of acute myeloid leukaemia. Lancet Oncol. 2006, 7, 350-352. [CrossRef]

94. Martínez-Losada, C.; Serrano-López, J.; Serrano-López, J.; Noguera, N.I.; Garza, E.; Piredda, L.; Lavorgna, S.; Consalvo, M.A.I.; Ottone, T.; Alfonso, V.; et al. Clonal genetic evolution at relapse of favorable-risk acute myeloid leukemia with NPM1 mutation is associated with phenotypic changes and worse outcomes. Haematologica 2018, 103, e400-e403. [CrossRef] [PubMed]

95. Krönke, J.; Bullinger, L.; Teleanu, V.; Tschürtz, F.; Gaidzik, V.I.; Kühn, M.W.; Rücker, F.G.; Holzmann, K.; Paschka, P.; Kapp-Schwörer, S.; et al. Clonal evolution in relapsed NPM1-mutated acute myeloid leukemia. Blood 2013, 122, 100-108. [CrossRef] [PubMed]

96. Bacher, U.; Porret, N.; Joncourt, R.; Sanz, J.; Aliu, N.; Wiedemann, G.; Jeker, B.; Banz, Y.; Pabst, T. Pitfalls in the molecular follow-up of NPM1 mutant acute myeloid leukemia. Haematologica 2018, 103, e486-e488. [CrossRef] [PubMed]

97. Pinheiro, L.B.; Coleman, V.A.; Hindson, C.M.; Herrmann, J.; Hindson, B.J.; Bhat, S.; Emslie, K.R. Evaluation of a droplet digital polymerase chain reaction format for DNA copy number quantification. Anal. Chem. 2011, 84, 1003-1011. [CrossRef] [PubMed]

98. Wertheim, G.B.W.; Bagg, A. NPM1 for MRD: Droplet like it's hot! J. Mol. Diagn 2017, 19, 498-501. [CrossRef] [PubMed]

99. Barakat, F.H.; Luthra, R.; Yin, C.C.; Barkoh, B.A.; Hai, S.; Jamil, W.; Bhakta, Y.I.; Chen, S.; Medeiros, L.J.; Zuo, Z. Detection of nucleophosmin 1 mutations by quantitative real-time polymerase chain reaction versus capillary electrophoresis: A comparative study. Arch. Pathol. Lab. Med. 2011, 135, 994-1000. [CrossRef] [PubMed]

100. Behjati, S.; Tarpey, P.S. What is next generation sequencing? Arch. Dis. Child. Educ. Pract. Ed. 2013, 98, 236-238. [CrossRef] [PubMed]

101. Yan, B.; Hu, Y.; Ban, K.H.K.; Tiang, Z.; Ng, C.; Lee, J.; Tan, W.; Chiu, L.; Wee Tan, T.; Seah, E.; et al. Single-cell genomic profiling of acute myeloid leukemia for clinical use: A pilot study. Oncol. Lett. 2017, 13, 1625-1630. [CrossRef] [PubMed]

102. Povinelli, B.J.; Rodriguez-Meira, A.; Mead, A.J. Single cell analysis of normal and leukemic hematopoiesis. Mol. Asp. Med. 2018, 59, 85-94. [CrossRef] [PubMed]

103. Zhu, S.; Qing, T.; Zheng, Y.; Jin, L.; Shi, L. Advances in single-cell RNA sequencing and its applications in cancer research. Oncotarget 2017, 8, 53763-53779. [CrossRef] [PubMed]

104. Luthra, R.; Patel, K.P.; Reddy, N.G.; Haghshenas, V.; Routbort, M.J.; Harmon, M.A.; Barkoh, B.A.; Kanagal-Shamanna, R.; Ravandi, F.; Cortes, J.E.; et al. Next-generation sequencing-based multigene mutational screening for acute myeloid leukemia using MiSeq: Applicability for diagnostics and disease monitoring. Haematologica 2014, 99, 465-473. [CrossRef] [PubMed]

105. Thol, F.; Gabdoulline, R.; Liebich, A.; Klement, P.; Schiller, J.; Kandziora, C.; Hambach, L.; Stadler, M.; Koenecke, C.; Flintrop, M.; et al. Measurable residual disease (MRD) monitoring by NGS before allogeneic hematopoietic cell transplantation in AML. Blood 2018, 132, 1703-1713. [CrossRef] [PubMed]

106. Klco, J.M.; Miller, C.A.; Griffith, M.; Petti, A.; Spencer, D.H.; Ketkar-Kulkarni, S.; Wartman, L.D.; Christopher, M.; Lamprecht, T.L.; Helton, N.M.; et al. Association between mutation clearance after induction therapy and outcomes in acute myeloid leukemia. JAMA 2015, 314, 811-822. [CrossRef] [PubMed]

107. Hirsch, P.; Tang, R.; Abermil, N.; Flandrin, P.; Moatti, H.; Favale, F.; Suner, L.; Lorre, F.; Marzac, C.; Fava, F.; et al. Precision and prognostic value of clone-specific minimal residual disease in acute myeloid leukemia. Haematologica 2017, 102, 1227-1237. [CrossRef] [PubMed]

108. Rothenberg-Thurley, M.; Amler, S.; Goerlich, D.; Köhnke, T.; Konstandin, N.P.; Schneider, S.; Sauerland, M.C.; Herold, T.; Hubmann, M.; Ksienzyk, B.; et al. Persistence of pre-leukemic clones during first remission and risk of relapse in acute myeloid leukemia. Leukemia 2018, 32, 1598-1608. [CrossRef] [PubMed]

109. Morita, K.; Kantarjian, H.P.; Wang, F.; Yan, Y.; Bueso-Ramos, C.; Sasaki, K.; Issa, G.C.; Wang, S.; Jorgensen, J.; Song, X.; et al. Clearance of somatic mutations at remission and the risk of relapse in acute myeloid leukemia. J. Clin. Oncol. 2018, 36, 1788-1797. [CrossRef] [PubMed]

110. Kayser, S.; Schlenk, R.F.; Grimwade, D.; Yosuico, V.E.D.; Walter, R.B. Minimal residual disease-directed therapy in acute myeloid leukemia. Blood 2015, 125, 2331-2335. [CrossRef] [PubMed] 
111. Kunchala, P.; Kuravi, S.; Jensen, R.; McGuirk, J.; Balusu, R. When the good go bad: Mutant NPM1 in acute myeloid leukemia. Blood Rev. 2018, 32, 167-183. [CrossRef] [PubMed]

112. Brunetti, L.; Gundry, M.C.; Sorcini, D.; Guzman, A.G.; Huang, Y.H.; Ramabadran, R.; Gionfriddo, I.; Mezzasoma, F.; Milano, F.; Nabet, B.; et al. Mutant NPM1 maintains the leukemic state through HOX expression. Cancer Cell 2018, 34, 499-512. [CrossRef] [PubMed]

113. Uckelmann, H.J.; Armstrong, S.A.; Stone, R.M. Location, location, location: Mutant NPM1c cytoplasmic localization is required to maintain stem cell genes in AML. Cancer Cell 2018, 34, 355-357. [CrossRef] [PubMed]

(c) (C) 2018 by the authors. Licensee MDPI, Basel, Switzerland. This article is an open access article distributed under the terms and conditions of the Creative Commons Attribution (CC BY) license (http://creativecommons.org/licenses/by/4.0/). 